\title{
Gobierno Móvil en Argentina: Análisis de Experiencia de Usuarios Basado en Comentarios en Tiendas de Aplicaciones
}

\section{Mobile Government in Argentina: User Experience Analysis Based on Comments in Application Stores}

\author{
Alejandra Carrizo, Gabriela Gaetan \\ acarrizo@uaco.unpa.edu.ar,ggaetan@uaco.unpa.edu.ar \\ Universidad Nacional de la Patagonia Austral (UNPA) \\ Unidad Académica Caleta Olivia (UACO) - Instituto de Tecnología Aplicada (ITA)
}

Recibido: 26/06/2019. Aceptado: 09/12/2019

\begin{abstract}
RESUMEN
Para promover el uso de los servicios del gobierno en cualquier momento y desde cualquier lugar, el gobierno móvil debe ser útil y fácil de usar; es decir, proporcionar una experiencia de usuario (UX) que satisfaga las necesidades de todos los ciudadanos. Analizar la UX permite conocer y evaluar cómo se sienten los usuarios con respecto a un producto, en función de facilidad de uso, valor percibido, utilidad, eficiencia, entre otros. Una opción para hacer este análisis es revisar fuentes de información existentes, tales como comentarios de usuarios en las tiendas de aplicaciones. El objetivo de este trabajo es determinar deficiencias y problemas que podrían afectar negativamente la experiencia de los ciudadanos al interactuar con aplicaciones de gobierno móvil en Argentina. Para este propósito, se recolectaron de la web datos sobre aplicaciones de gobierno móvil en Argentina |, y se analizaron los comentarios creando un Diagrama de Afinidad basado en las características del modelo ISO/IEC 25010. El análisis de resultados permitió identificar aspectos que pueden ayudar a mejorar la experiencia de los ciudadanos. El hallazgo más relevante que surgió es que los usuarios reclaman aplicaciones útiles y beneficiosas, con información actualizada y servicios accesibles en cualquier momento y lugar. También se observó que las aplicaciones no son actualizadas frecuentemente y que es escasa su promoción desde los sitios web y redes sociales institucionales.
\end{abstract}

Palabras clave: Gobierno Móvil; Experiencia de Usuario; Usabilidad; Accesibilidad Web.

\begin{abstract}
To promote the use of government services at any time and from anywhere, mobile government must be useful and easy to use; that is, provide a user experience (UX) that meets the needs of all citizens. Analyzing the UX allows us to know and evaluate how users feel about a product, based on ease of use, perceived value, utility, efficiency, among others. One option for doing this analysis is to review existing information sources, such as user comments in the application stores. The objective of this work is to identify deficiencies and problems that could negatively affect the experience of citizens when interacting with mobile government applications in Argentina. For this purpose, data on mobile government applications in Argentina were collected from the web, and the comments were analyzed creating an Affinity Diagram based on the characteristics of the ISO / IEC 25010 model. The analysis of results allowed identifying aspects that can help improve the experience of
\end{abstract}


citizens. The most relevant finding that emerged is that users claim useful and beneficial applications, with updated information and accessible services at any time and place. It was also observed that the applications are not updated frequently and that their promotion is scarce from the websites and institutional social networks.

Key words: Mobile Government; User Experience; Usability; Web Accessibility.

\section{INTRODUCCIÓN}

El concepto de gobierno electrónico se refiere a la aplicación de las TIC (Tecnologías de la Información y de la Comunicación) en los órganos de la Administración para mejorar la información y los servicios ofrecidos a los ciudadanos (CLAD, 2007). El gobierno móvil es una área específica del gobierno electrónico. Por medio del Gobierno móvil se habilita a ciudadanos, negocios y empleados públicos con mejores accesos a información y servicios vía tecnología móvil (Yu, 2014) y (ZamZami, Mahmud, \& Abubakar, 2014).

El gobierno móvil promueve el uso de aplicaciones móviles para brindar servicios a los ciudadanos en modo transparente, eficaz y eficiente. Y por su parte, los ciudadanos esperan acceder a estos servicios de manera fácil y amigable. En consecuencia, estas aplicaciones móviles deben proporcionar experiencias de usuario que cumplan con las necesidades de los distintos usuarios.

Por su parte, el desarrollo de Experiencia de Usuario (UX, del inglés User eXperience) implica especificar productos enfocados en las personas que los usan y las tareas que esas personas llevan a cabo (Hartson \& Pyla, 2012). En particular, la UX móvil no solo tiene en cuenta al usuario, sino también cómo utiliza el dispositivo, cuándo lo consulta y cómo navega por la aplicación.

En Argentina, según el informe Acceso y Uso de Tecnologías de la Información y la Comunicación (Instituto Nacional de Estadística y Censos, 2019), en el cuarto trimestre de $2018,83,5 \%$ de la población utiliza teléfono celular. En ese contexto, nuestra investigación está enfocada en determinar deficiencias y problemas que podrían afectar negativamente la experiencia de los ciudadanos al interactuar con aplicaciones de Gobierno móvil en Argentina.

Luego de una revisión de las aplicaciones móviles disponibles, este trabajo analiza la UX en base a información publicada en la web; principalmente, en las opiniones que los usuarios publican en tiendas de aplicaciones. Para esto, recopilamos de la web datos relevantes sobre aplicaciones de Gobierno móvil en Argentina y los organizamos en base a la estructura planteada en trabajos anteriores (Carrizo, Gaetán, Saldaño, \& Martin, 2018); luego, realizamos un Diagrama de Afinidad con los comentarios recolectados, agrupándolos y etiquetando los grupos según las características del modelo de calidad ISO/IEC 25010. A partir de los resultados obtenidos, identificamos algunos problemas que afectan negativamente a la Experiencia de usuario con esas aplicaciones.

Este documento se ha estructurado de la siguiente manera: La Sección 1 se enfoca en la experiencia de usuario (UX) móvil, y en la Investigación de Experiencia de Usuario. En la Sección 2, se detalla el modelo de calidad ISO/IEC 25010. Mientras que en la Sección 3 se aborda el tema de Gobierno móvil. Por su parte, en la Sección 4 se describen los Materiales y 
Métodos de este estudio, y los Resultados en la Sección 5. Finalmente, se desarrollan las conclusiones.

\section{MARCO TEORICO}

\section{Experiencia de Usuario}

La Experiencia del Usuario (Hartson \& Pyla, 2012) es el proceso de mejorar la satisfacción con un producto al mejorar la usabilidad, la accesibilidad y la satisfacción proporcionados en la interacción con ese producto.

Por un lado, la usabilidad refleja facilidad y eficiencia del uso, y es una parte importante de la experiencia del usuario y, por lo tanto, de la satisfacción del usuario. El estándar ISO 9241 (ISO/TC 159/SC 4, 2018) define usabilidad como "el grado de efectividad, eficiencia y satisfacción con el que los usuarios especificados pueden alcanzar objetivos específicos en contextos específicos de uso". Mientras que (Nielsen J. , 1999), define usabilidad como "un atributo cualitativo que evalúa el grado de usabilidad de una interfaz y se refiere proactivamente a los métodos que pueden usarse para mejorar dicha facilidad de uso durante el proceso de diseño".

Por otro lado, Accesibilidad Web, como explica el (W3C-WAI), "significa que las personas con discapacidad pueden percibir, comprender, navegar e interactuar con la Web y que pueden contribuir a la Web"; esto se aplica también a las personas con cualquier tipo de discapacidad temporal o permanente.

En particular, el diseño de UX para móviles se enfoca principalmente en la eficiencia y la capacidad de descubrimiento (en inglés, discoverability). En (Nielsen \& Budiu, 2015) se describe un conjunto de componentes que forman la UX móvil, como una herramienta conceptual para construir y evaluar buenas experiencias móviles, dentro del contexto de un enfoque centrado en el usuario:

- Funcionalidad. Incluye herramientas y características que permiten a los usuarios completar tareas y alcanzar sus objetivos.

- Arquitectura de Información. Se refiere a la forma de organizar funcionalidad y contenido en una estructura lógica, para ayudar a los usuarios a encontrar información y completar tareas. Esto incluye navegación, búsqueda y etiquetado.

- Contenido Incluye contenido en diferentes formatos, como texto, imágenes y video, que proporcionan información al usuario.

- Diseño. Se refiere a la presentación visual y la experiencia interactiva de dispositivos móviles, incluidos diseño gráfico, marca y diseño.

- Entrada del usuario. Se enfoca en el esfuerzo requerido para ingresar datos, que debe minimizarse y no requerir el uso de ambas manos.

- Contexto móvil. Trata sobre el entorno y las circunstancias de uso, cualquier cosa que afecte la interacción entre usuario e interface.

- Usabilidad Es la medida general de qué tan bien la arquitectura de información, el diseño, el contenido y otros elementos trabajan en conjunto para permitir a los usuarios lograr sus objetivos.

- Integridad. Se relaciona con el nivel de confianza, seguridad y comodidad que los usuarios sienten al usar un sitio web o aplicación móvil. 
- Realimentación. Son métodos para atraer la atención del usuario y mostrar información importante.

- Ayuda. Son opciones, productos y servicios que están disponibles para ayudar al usuario a usar el sitio web o la aplicación.

- Social. Se relaciona el contenido y las características que crean un sentido de participación social, que permiten la interacción del usuario y que facilitan el intercambio en las redes sociales establecidas.

- Marketing. Se refiere a los métodos por los cuales un usuario encuentra un sitio web o aplicación y los factores que fomentan el uso repetido.

\subsection{Características de los Usuarios Móviles}

De acuerdo a lo que expresa (Ballard, 2007), en cierto punto no existe una diferencia particular entre los usuarios de dispositivos móviles y los usuarios de otros dispositivos. La autora resalta que la diferencia principal con los usuarios de computadoras es precisamente la movilidad, lo que determina las siguientes características de los usuarios de aplicaciones móviles:

- Móvil. Un usuario que se conecta a través de un dispositivo móvil, por lo general, consume el contenido por un tiempo mucho más corto que cuando lo hace a través de computadoras.

- Fácil de interrumpir y distraer. El usuario móvil tiene las mismas fuentes de interrupción del mundo físico que tiene el usuario de escritorio, pero carece de las señales que sugieren que no está disponible para la interrupción. En la mayoría de los casos, su mera presencia en un espacio social público podría indicar que es interrumpible.

- Disponible. El lado inverso a la interrumpibilidad es que los usuarios de teléfonos móviles están disponibles rápidamente para sus contactos. La mayoría de los dispositivos de comunicación personal están con el usuario constantemente, principalmente en las partes relevantes del día.

- Sociable. Los usuarios de dispositivos móviles también tienen relación con las personas que los rodean. Los comportamientos sociales variarán en función de quién está físicamente presente, dónde está la presencia, el estado de ánimo actual, el tipo de comunicación y la fuente de comunicación entrante.

- Contextual. El entorno del usuario móvil afecta cómo se usa el dispositivo. Los dispositivos podrían hacer uso de sensores de aceleración, sensores de temperatura, lectores de huellas dactilares y otras fuentes de información que determinan el contexto en el que se encuentra el usuario.

- Identificable. Ya que los dispositivos son personales, generalmente son únicamente para un usuario. Esta identificación incluye tanto la dirección de mensajería única como el dispositivo.

\subsection{Investigación de Experiencia de Usuario}

La Investigación de Experiencia de Usuario (en inglés, User Experience Research) es el proceso de descubrir los comportamientos, motivaciones y necesidades de los clientes a través de observación, análisis de tareas y otros tipos de comentarios de los usuarios.

El campo de UX tiene una amplia gama de métodos de investigación disponibles; como se muestra en la Tabla 1, para decidir cuál es el mejor método para cada proyecto se consideran tres dimensiones de investigación. 
Tabla 1. Dimensiones de Investigación de experiencia de usuario.

\begin{tabular}{|l|l|}
\hline Dimensión & Características \\
\hline Actitudinal vs. & $\begin{array}{l}\text { Actitudinal se refiere a lo que los usuarios dicen, mientras que conductual se } \\
\text { refiere a lo que realmente hacen. La investigación actitudinal se usa a menudo en } \\
\text { marketing porque mide las creencias y necesidades expresadas por las personas. } \\
\text { Sin embargo, lo que usuarios realmente hacen es más relevante en el diseño. }\end{array}$ \\
\hline $\begin{array}{l}\text { Cuantitativo vs. } \\
\text { Cualitativo }\end{array}$ & $\begin{array}{l}\text { Los estudios que son de naturaleza cualitativa generan datos sobre } \\
\text { comportamientos o actitudes basados en observación directa, mientras que en } \\
\text { estudios cuantitativos, los datos sobre el comportamiento o las actitudes en } \\
\text { cuestión se recopilan de manera indirecta, a través de una medición o un } \\
\text { instrumento, como una encuesta o una herramienta analítica. El análisis } \\
\text { cualitativo de los datos no suele ser matemático; mientras que el conocimiento en } \\
\text { los métodos cuantitativos se derivan típicamente del análisis matemático, ya que } \\
\text { el instrumento de recopilación de datos captura grandes cantidades de datos que } \\
\text { son fácilmente codificados numéricamente. }\end{array}$ \\
\hline Contexto de uso & $\begin{array}{l}\text { Al recopilar y analizar información sobre los usuarios, el uso previsto del } \\
\text { producto, y las tareas que realizan los usuarios con el producto, el análisis del } \\
\text { contexto de uso permite a los investigadores comprender mejor la experiencia del } \\
\text { usuario. Por lo general, los datos del análisis de contexto de uso se recopilan a } \\
\text { través de encuestas, entrevistas, visitas a sitios, grupos focales y estudios de } \\
\text { observación. Este tipo de investigación generalmente se realiza al principio del } \\
\text { ciclo de vida del producto y continúa a medida que los datos identifican qué } \\
\text { componentes del producto y la experiencia del usuario son más críticos. }\end{array}$ \\
\hline
\end{tabular}

\subsubsection{Métodos de Investigación de Experiencia de Usuario}

Existen diversos tipos de investigación de UX disponibles, algunos métodos se utilizan para comprender cómo piensan y sienten los usuarios, qué necesitan y desean, y por qué. Por ejemplo, las entrevistas, las encuestas y la clasificación de tarjetas (Cardsorting). Otros métodos se usan para analizar y entender el comportamiento del usuario, como el Testing de usabilidad, observación in-site, o revisiones analíticas.

Otro grupo de métodos de investigación de UX no implica un estudio directo de los usuarios, sino más bien una evaluación del panorama y de elementos específicos existentes de la experiencia del usuario. Entre ellos se puede mencionar:

Revisión Competitiva. La revisión competitiva se realiza típicamente como un estudio cualitativo de una pequeña muestra de competidores directos e indirectos. Los competidores directos son compañías que ofrecen la misma propuesta de valor, o una muy similar, al mismo segmento de clientes que atiende nuestro cliente. Los competidores indirectos son compañías que ofrecen una propuesta de valor similar a un segmento de clientes diferente del que atiende nuestro cliente.

Auditoria de Contenido. Una auditoría de contenido se realiza como un método de investigación cualitativo y evaluativo que puede emplearse para comprender mejor el estado actual de un producto existente. Es un proceso de creación y evaluación de un inventario de todos los contenidos y activos en un sitio web, incluida la grabación de la estructura del contenido y las relaciones entre los bloques de contenido. También puede incluir un análisis 
del vocabulario utilizado como parte de la interfaz de usuario para evaluar su calidad y consistencia.

Revisión de Experiencia de Usuario. Una revisión de la experiencia del usuario de un sitio web o aplicación existente es un método de evaluación cualitativo que permite a los revisores analizar el estado actual a través de uno de los siguientes enfoques:

- Evaluación heurística. Es un método para encontrar problemas de usabilidad en un producto de software revisando el cumplimiento de una lista de principios de usabilidad reconocidos llamados heurísticos. Es realizado por un grupo de evaluadores capacitados que evalúan individualmente un producto y luego combinan sus resultados para llegar a una lista priorizada de problemas. Expertos en usabilidad y accesibilidad han compilado listas de principios para ser aplicadas al realizar análisis heurísticos. Entre ellas se encuentran las "10 heurísticas de usabilidad para el diseño de interfaz de usuario" (Nielsen J. , 10 usability heuristics for user interface design, 1995), que son probablemente el conjunto de heurísticas de usabilidad más comúnmente utilizado. Hay otros, como la "Lista de los Seis Principios de Diseño para la Usabilidad" (Norman, 2013), y las "247 Pautas de Usabilidad Web" de Travis (Travis, 2009).

- Revisión de expertos. Este método no depende estrictamente de un conjunto estándar de heurísticas. El experto puede ampliar o limitar el conjunto de mejores prácticas en las que se basa la revisión. Por ejemplo, una de las Heurísticas de Nielsen estipula como buena práctica el "Diseño estético y minimalista"; sin embargo en el caso de los juegos, la experiencia depende en gran medida de una estética muy rica y no minimalista. Una revisión de expertos ofrece más flexibilidad que una evaluación heurística. El énfasis en la experiencia del revisor y el conocimiento de los principios de usabilidad es la razón por la cual este tipo de revisión se conoce como revisión experta.

\section{Modelo de Calidad ISO/IEC 25010}

Un modelo de calidad puede ser concebido como el conjunto de factores de calidad, y de las relaciones entre ellos, que suministran un soporte para la especificación y evaluación de la calidad. El modelo de calidad ISO/IEC 25010 (ISO/IEC JTC 1/SC 7, 2011) describe dos perspectivas de calidad: producto y en uso. La perspectiva de la calidad del producto se relaciona con el diseño del producto central (características internas y externas), mientras que el aspecto de la calidad en uso se relaciona con la interacción del usuario con un producto en un contexto de uso específico. ISO/IEC 25010, por lo tanto, puede utilizarse potencialmente para modelar UX.

El modelo de calidad del producto ISO/IEC 25010 está compuesto por 8 características de calidad, y una serie de subcaracterísticas que a continuación se detallan:

1. Adecuación Funcional. Representa la capacidad del producto software para proporcionar funciones que satisfacen las necesidades declaradas e implícitas, cuando el producto se usa en las condiciones especificadas.

1.1. Completitud funcional. Grado en el cual el conjunto de funcionalidades cubre todas las tareas y los objetivos del usuario especificados.

1.2. Corrección funcional. Capacidad del producto o sistema para proveer resultados correctos con el nivel de precisión requerido.

1.3. Pertinencia funcional. Capacidad del producto software para proporcionar un conjunto apropiado de funciones para tareas y objetivos de usuario especificados. 
2. Eficiencia de desempeño. Esta característica representa el desempeño relativo a la cantidad de recursos utilizados bajo determinadas condiciones.

2.1. Comportamiento temporal. Los tiempos de respuesta y procesamiento y los ratios de throughput de un sistema cuando lleva a cabo sus funciones bajo condiciones determinadas en relación con un banco de pruebas (benchmark) establecido.

2.2. Utilización de recursos. Las cantidades y tipos de recursos utilizados cuando el software lleva a cabo su función bajo condiciones determinadas.

2.3. Capacidad. Grado en que los límites máximos de un parámetro de un producto o sistema software cumplen con los requisitos.

3. Compatibilidad. Capacidad de dos o más sistemas o componentes para intercambiar información y/o llevar a cabo sus funciones requeridas cuando comparten el mismo entorno hardware o software.

3.1. Coexistencia. Capacidad del producto para coexistir con otro software independiente, en un entorno común, compartiendo recursos comunes sin detrimento.

3.2. Interoperabilidad. Capacidad de dos o más sistemas o componentes para intercambiar información y utilizar la información intercambiada.

4. Usabilidad. Capacidad del producto software para ser entendido, aprendido, usado y resultar atractivo para el usuario, cuando se usa bajo determinadas condiciones.

4.1. Capacidad para reconocer su adecuación. Capacidad del producto que permite al usuario entender si el software es adecuado para sus necesidades.

4.2. Capacidad de aprendizaje. Capacidad del producto que permite al usuario aprender su aplicación.

4.3. Capacidad para ser usado. Capacidad del producto que permite al usuario operarlo y controlarlo con facilidad.

4.4. Protección contra errores de usuario. Capacidad del sistema para proteger a los usuarios de hacer errores.

4.5. Estética de la interfaz de usuario. Capacidad de la interfaz de usuario de agradar y satisfacer la interacción con el usuario.

4.6. Accesibilidad. Capacidad del producto que permite que sea utilizado por usuarios con determinadas características y discapacidades.

5. Fiabilidad. Capacidad de un sistema o componente para desempeñar las funciones especificadas, cuando se usa bajo unas condiciones y periodo de tiempo determinados.

5.1. Madurez. Capacidad del sistema para satisfacer las necesidades de fiabilidad en condiciones normales.

5.2. Disponibilidad. Capacidad del sistema o componente de estar operativo y accesible para su uso cuando se requiere.

5.3. Tolerancia a fallos. Capacidad del sistema o componente para operar según lo previsto en presencia de fallos hardware o software.

5.4. Capacidad de recuperación. Capacidad del producto software para recuperar los datos directamente afectados y restablecer el estado deseado del sistema en caso de interrupción o fallo.

6. Seguridad. Capacidad de protección de la información y los datos de manera que personas o sistemas no autorizados no puedan leerlos o modificarlos.

6.1. Confidencialidad. Capacidad de protección contra el acceso de datos e información no autorizados, ya sea accidental o deliberadamente.

6.2. Integridad. Capacidad del sistema o componente para prevenir accesos o modificaciones no autorizados a datos o programas de ordenador.

6.3. No repudio. Capacidad de demostrar las acciones o eventos que han tenido lugar, de manera que dichas acciones o eventos no puedan ser repudiados posteriormente. 
6.4. Responsabilidad. Capacidad de rastrear de forma inequívoca las acciones de una entidad.

6.5. Autenticidad. Capacidad de demostrar la identidad de un sujeto o un recurso.

7. Mantenibilidad. Esta característica representa la capacidad del producto software para ser modificado efectiva y eficientemente, debido a necesidades evolutivas, correctivas o perfectivas.

7.1. Modularidad. Capacidad de un sistema o programa de ordenador que permite que un cambio en un componente tenga un impacto mínimo en los demás.

7.2. Reusabilidad. Capacidad de un activo que permite que sea utilizado en más de un sistema software o en la construcción de otros activos.

7.3. Analizabilidad. Facilidad con la que se puede evaluar el impacto de un determinado cambio sobre el resto del software, diagnosticar las deficiencias o causas de fallos en el software, o identificar las partes a modificar.

7.4. Capacidad para ser modificado. Capacidad del producto que permite que sea modificado de forma efectiva y eficiente sin introducir defectos o degradar el desempeño.

7.5. Capacidad para ser probado. Facilidad con la que se pueden establecer criterios de prueba para un sistema o componente y con la que se pueden llevar a cabo las pruebas para determinar si se cumplen dichos criterios.

8. Portabilidad. Capacidad del producto o componente de ser transferido de forma efectiva y eficiente de un entorno hardware, software, operacional o de utilización a otro.

8.1. Adaptabilidad. Capacidad del producto que le permite ser adaptado de forma efectiva y eficiente a diferentes entornos determinados de hardware, software, operacionales o de uso.

8.2. Capacidad para ser instalado. Facilidad con la que el producto se puede instalar y/o desinstalar de forma exitosa en un determinado entorno.

8.3. Capacidad para ser reemplazado. Capacidad del producto para ser utilizado en lugar de otro producto software determinado con el mismo propósito y en el mismo entorno.

Por su parte, el modelo de calidad de uso es el grado en que un producto o sistema puede ser utilizado por usuarios específicos para satisfacer sus necesidades de lograr objetivos específicos con efectividad, eficiencia, ausencia de riesgo y satisfacción en contextos específicos de uso. Las propiedades de este modelo se clasifican en 5 características:

1. Eficacia. Precisión e integridad con la que los usuarios logran objetivos específicos.

2. Eficiencia. Recursos gastados en relación con la precisión y la integridad con que los usuarios logran los objetivos.

3. Satisfacción. El grado en que las necesidades de los usuarios se satisfacen cuando un producto o sistema se utiliza en un contexto específico de uso.

3.1. Utilidad. El grado en que un usuario está satisfecho con el logro percibido de los objetivos pragmáticos, incluidos los resultados del uso y las consecuencias del uso.

3.2. Confianza. El grado en que un usuario u otra parte interesada tiene confianza en que un producto o sistema se comportará según lo previsto.

3.3. Placer. Grado en que un usuario obtiene placer al satisfacer sus necesidades personales.

3.4. Comodidad. Grado de satisfacción del usuario con el confort físico.

4. Ausencia de riesgo. El grado en que un producto o sistema mitiga el riesgo potencial para el estado económico, la vida humana, la salud o el medio ambiente. 
4.1. Mitigación del riesgo económico. El grado en que un producto o sistema mitiga el riesgo potencial para el estado financiero, la operación eficiente, la propiedad comercial, la reputación u otros recursos en los contextos de uso previstos.

4.2. Mitigación de riesgos para la salud y la seguridad. El grado en que un producto o sistema mitiga el riesgo potencial para las personas en los contextos de uso previstos.

4.3. Mitigación de riesgos ambientales. El grado en que un producto o sistema mitiga el riesgo potencial para la propiedad o el medio ambiente en los contextos de uso previstos.

5. Cobertura de contexto. El grado en que un producto o sistema se puede usar con efectividad, eficiencia, libertad de riesgo y satisfacción tanto en contextos específicos de uso como en contextos más allá de los identificados inicialmente de manera explícita.

5.1. Integridad del contexto. El grado en que un producto o sistema se puede usar con efectividad, eficiencia, libertad de riesgo y satisfacción en todos los contextos específicos de uso.

5.2. Flexibilidad. El grado en que un producto o sistema puede usarse con efectividad, eficiencia, libertad de riesgo y satisfacción en contextos más allá de los inicialmente especificados en los requisitos.

\section{Gobierno Electrónico}

Tal como se plantea en (Naser \& Concha, 2011), no existe una única definición de Gobierno Electrónico; la OCDE (Organización para la Cooperación y el Desarrollo Económicos) lo definió originalmente como "la aplicación de tecnologías basadas en Internet para actividades comerciales y no comerciales en el seno de las administraciones públicas", y luego como "el uso de las tecnologías de la información y comunicación (TIC), particularmente la Internet, como una herramienta para alcanzar un mejor gobierno".

De acuerdo a la Carta Iberoamericana de Gobierno Electrónico (CLAD, 2007), se entienden las expresiones de Gobierno Electrónico y de Administración Electrónica como sinónimos; ambas consideran al uso de las Tecnologías de la Información y Comunicación en los órganos de la administración para mejorar la información y los servicios ofrecidos a los ciudadanos, aumentan la eficacia y eficiencia de la gestión pública e incrementan sustantivamente la transparencia del sector y la participación de los ciudadanos. La Tabla 2 resume, de acuerdo a este mismo documento, los principios que inspiran el Gobierno Electrónico.

Tabla 2. Principios del Gobierno electrónico.

\begin{tabular}{|l|l|}
\hline Principio & Descripción \\
\hline Igualdad & $\begin{array}{l}\text { El uso de medios electrónicos no implica restricciones para ciudadanos que se } \\
\text { relacionen con Administraciones Públicas por medios no electrónicos. }\end{array}$ \\
\hline Legalidad & $\begin{array}{l}\text { Las garantías previstas en los modos tradicionales de relación del ciudadano } \\
\text { con el Gobierno y la Administración se mantienen idénticas en los medios } \\
\text { electrónicos. Este principio comprende el respeto a la privacidad. }\end{array}$ \\
\hline $\begin{array}{l}\text { Conservación } \\
\text { accesibilidad }\end{array}$ & $\begin{array}{l}\text { Las comunicaciones y documentos electrónicos se conservan en las mismas } \\
\text { condiciones que por los medios tradicionales. }\end{array}$ \\
\hline Proporcionalidad & $\begin{array}{l}\text { La información y el conocimiento de servicios por medios electrónicos se } \\
\text { realizan en un lenguaje comprensible según el perfil del destinatario. }\end{array}$ \\
\hline Responsabilidad & $\begin{array}{l}\text { Los requerimientos de seguridad deben ser adecuados a la naturaleza de la } \\
\text { relación que se establezca con la Administración. }\end{array}$ \\
\hline
\end{tabular}




\begin{tabular}{|l|l|}
\hline & electrónicos de la misma manera que de los realizados por medios tradicionales. \\
\hline $\begin{array}{l}\text { Adecuación } \\
\text { tecnológica }\end{array}$ & $\begin{array}{l}\text { Las administraciones elegirán las tecnologías más adecuadas para satisfacer sus } \\
\text { necesidades. Se recomienda el uso de estándares abiertos y de software libre en } \\
\text { razón de la seguridad, sostenibilidad a largo plazo y para prevenir que el } \\
\text { conocimiento público no sea privatizado. }\end{array}$ \\
\hline
\end{tabular}

El Gobierno Electrónico contribuye a la gestión de tal forma que genera un gobierno ágil, transparente, eficaz, eficiente, productivo, participativo y proactivo, capaz de promover un desarrollo local sostenible.

\subsection{Gobierno Móvil}

El concepto de Gobierno móvil se basa en el de Gobierno electrónico o e-gobierno. Tanto (Al-Nuaim, 2014) como (Costopoulou \& Molhanec, 2014) coinciden en que el gobierno móvil es una extensión del gobierno digital que utiliza aplicaciones inalámbricas $o$ plataformas móviles para teléfonos o dispositivos inteligentes. Mientras que (Yu, 2014) y (ZamZami, Mahmud, \& Abubakar, 2014) sugieren que gobierno móvil es una extensión no sólo técnica sino también funcional del gobierno digital para habilitar a ciudadanos, negocios y empleados públicos con mejores accesos a información y servicios vía tecnología móvil.

Entonces, para los usuarios, el Gobierno móvil puede ayudar a que la información y los servicios gubernamentales resulten accesibles en cualquier momento y lugar. Por medio del Gobierno móvil, se promueve eficiencia en la prestación de ciertos servicios y una ciudadanía más activa a través de información, requerimientos o reclamos instantáneos. El Gobierno móvil se puede ver como un complemento para el e-gobierno, ya que si bien los dispositivos móviles son excelentes vías de acceso, cuando se trata de transmitir información compleja o voluminosa, la mayoría de ellos no son adecuados.

De acuerdo a (OECD/International Telecommunication Union , 2011), la importancia del Gobierno móvil resulta crucial para obtener una serie de ventajas o beneficios para los distintos tipos de usuarios existentes:

1) El Gobierno móvil puede ayudar a fortalecer los buenos resultados y generar una sociedad más conectada, mejorando la provisión de servicios por parte de las Administraciones Públicas.

2) El Gobierno móvil puede dotar de capacidades transformativas e innovadoras a las Administraciones Públicas. Es capaz de empoderar a aquellos ciudadanos postergados por la brecha digital, mejorando la calidad de vida de los afectados por la exclusión digital.

3) El Gobierno móvil puede intensificar las alianzas y el intercambio entre el sector público y privado. El avance de las tecnologías móviles está aportando mucho al desarrollo empresarial: existen mejoras en las plataformas tecnológicas para crear aplicaciones, que a su vez generan oportunidades para los ciudadanos.

\section{RESULTADOS, ANÁLISIS Y DISCUSIÓN}

\section{Materiales y Métodos}

El Proceso de Investigación de Experiencia de usuario que aplicamos en este estudio, es una adaptación de la propuesta de (Hall, 2013). A continuación describimos en detalle cada uno de los elementos que lo conforman. 


\subsection{Definición del Problema}

El objetivo principal de esta investigación fue determinar deficiencias y problemas que podrían afectar negativamente la experiencia de los ciudadanos al interactuar con aplicaciones de Gobierno Móvil en Argentina.

Luego de una revisión de las aplicaciones móviles relevantes, definimos como base de este estudio al conjunto de aplicaciones enumeradas en el sitio de aplicaciones móviles del Estado argentino $^{1}$. Si bien estas aplicaciones están disponibles tanto para Android como para iOS, el estudio se enfocó en aplicaciones para Android, ya que este sistema operativo, entre enero de 2018 y enero de 2019, representó 89,88\% del mercado en Argentina (Statcounter Global Stats $^{2}$.

\subsection{Selección del Enfoque}

Las opiniones de usuarios contienen información valiosa: reporte de errores, solicitud de características nuevas o mejoradas, explicación de la experiencia en el uso, entre otras (Iacob \& Harrison, 2013), (Galvis Carreño \& Winbladh, 2013). Teniendo en cuenta esto, en nuestro estudio, realizamos una investigación de experiencia de usuario mediada por Internet (Hewson \& Laurent, 2017), analizando opiniones de usuarios en la tienda de aplicaciones Google Play Store ${ }^{3}$.

\subsection{Recolección de Datos}

En el período del 1 de febrero al 30 de abril de 2019, recolectamos datos a los efectos de identificar problemas que podrían afectar negativamente la Experiencia de Usuario con aplicaciones móviles del Estado argentino. Para esta recolección, adaptamos la propuesta presentada en un trabajo anterior (Carrizo, Gaetán, Saldaño, \& Martin, 2018), y diseñamos la planilla de caracterización de aplicaciones de gobierno móvil, que se describe en la Tabla 3.

Tabla 3. Planilla de caracterización de aplicaciones de gobierno.

\begin{tabular}{|l|l|l|l|}
\hline Variable & Descripción & Fuente $^{(\mathbf{1})}$ & Ejemplo \\
\hline Aplicación & $\begin{array}{l}\text { Nombre de la } \\
\text { aplicación }\end{array}$ & Tienda & Aerolíneas móvil \\
\hline Valoración & $\begin{array}{l}\text { Promedio de } \\
\text { calificaciones de } \\
\text { usuarios }\end{array}$ & Tienda & 3,1 \\
\hline Opiniones & $\begin{array}{l}\text { Cantidad de opiniones } \\
\text { de usuarios }\end{array}$ & Tienda & 2.448 \\
\hline Actualización & $\begin{array}{l}\text { Fecha última } \\
\text { actualización }\end{array}$ & Tienda & 16 de abril de 2018 \\
\hline Descargas & Cantidad de descargas & Tienda & $100.000+$ \\
\hline Titular & Gobierno, Externo & Tienda & Gobierno \\
\hline Enlace & Enlace a tienda de & Sitio & www.aerolineas.com.ar/es- \\
\hline
\end{tabular}

\footnotetext{
${ }^{1}$ Portal Oficial del Estado Argentino. Aplicaciones móviles. https://www.argentina.gob.ar/aplicaciones-moviles

2 StatCounter. Mobile Mobile Operating System Market Share in Argentina - May 2019. http://gs.statcounter.com/os-market-share/mobile/argentina/\#monthly-201801-201901-bar

${ }^{3}$ Google Play Store. https://play.google.com/store
} 


\begin{tabular}{|l|l|l|l|}
\hline Institucional & aplicaciones & oficial & ar/aerolineas_movil \\
\hline Comentarios & $\begin{array}{l}\text { Texto del comentario, } \\
\text { fecha publicación, } \\
\text { valoración. }\end{array}$ & Tienda & $\begin{array}{l}* * * * 8 \text { de julio de 2018 } \\
\text { No dejajadiuntar fotos solo la a queja, ahora a esperar que por lo menos } 1 \text { qujaja sea respondi- } \\
\text { da }\end{array}$ \\
\hline
\end{tabular}

(1) Fuente de los datos: Tienda: https://play.google.com/store. Descarga Institucional: Sitio web oficial del organismo asociado o N/D (No Disponible, en caso que no se encuentre enlace para descargar desde el sitio institucional).

Por cada una de las aplicaciones enumeradas en el sitio de aplicaciones móviles del Estado argentino, accedimos a la tienda de aplicaciones Google Play Store, y registramos los datos que describe la Tabla 3. También verificamos la existencia de enlaces que permitieran descargar la aplicación desde el sitio oficial de la institución asociada y registramos este dato en la planilla.

Luego, para cada comentario que evidenciara problemas relacionados con la Experiencia de Usuario, registramos en la planilla: el texto del comentario, la fecha en que se publicó y la calificación que otorgó el usuario.

\subsubsection{Categorización de Problemas}

A fin de categorizar los problemas recurrentes que los usuarios de aplicaciones manifestaban en los comentarios de la tienda de descargas, aplicamos el método Diagrama de Afinidad:

1) Revisamos los comentarios registrados en la Planilla de caracterización de aplicaciones de gobierno (Tabla 3).

2) Copiamos el comentario en una nota adhesiva, indicando la aplicación de la que se trataba.

3) Identificamos patrones para agrupar las notas en una pizarra.

4) Etiquetamos los grupos identificados en base al modelo ISO/IEC 25010.

Como resultado, para categorizar los problemas manifestados en comentarios de los usuarios, identificamos un subconjunto de 13 características del modelo ISO/IEC 25010: Completitud funcional, Correctitud funcional, Interoperatividad, Eficiencia, Disponibilidad, Modificabilidad, Capacidad de instalación, Utilidad, Confianza, Confidencialidad, Aprendibilidad, Estética de interfaz de usuario, Accesibilidad.

\section{Resultados}

El sitio web del Gobierno argentino muestra 50 aplicaciones; sin embargo, este estudio se basó sólo en 31 aplicaciones, ya que, al momento de recolectar los datos, 17 de ellas no estaban disponibles, y 2 no registraban opiniones de los usuarios en la tienda de aplicaciones.

Los datos recolectados se registraron en la Planilla de caracterización de aplicaciones de gobierno. En la Tabla 4 se muestran los datos relacionados con la oferta de aplicaciones móviles del Gobierno argentino; mientras que los datos relacionados con los comentarios se ejemplifican en el Anexo I.

Luego, para identificar problemas de experiencia de usuario, exploramos estos datos desde dos perspectivas. Por un lado, analizamos los comentarios de usuarios, y por otro lado, las características generales de las aplicaciones ofertadas por el Gobierno Argentino (cantidad de descargas, valoración de los usuarios, cantidad de opiniones, medios de promoción, actualización y mantenimiento). 
Tabla 4. Registro de caracterización de aplicaciones de gobierno Móvil en Argentina.

\begin{tabular}{|c|c|c|c|c|c|c|}
\hline Aplicación & Valoración & Opiniones & Actualización & Descargas & Titular & Enlace Institucional ( ${ }^{(1)}$ \\
\hline Aerolíneas móvil & 3,1 & 2.448 & $16 / 04 / 2018$ & $100.000_{+}$ & Externos & https://www.aerolineas.com.ar/es-ar/aerolineas_movil \\
\hline Alertamos & 3,4 & 399 & $16 / 06 / 2016$ & $50.000+$ & Gobierno & http://alertamos.smn.gov.ar/ \\
\hline Argentina Salud & 3 & 10 & $06 / 08 / 2018$ & $1.000+$ & Gobierno & $\mathrm{N} / \mathrm{D}$ \\
\hline BA Subtes & 3,8 & 2.763 & $22 / 05 / 2018$ & $100.000+$ & Gobierno & https://www.buenosaires.gob.ar/aplicacionesmoviles/ba-subte \\
\hline BA Taxi & 3,1 & 554 & $13 / 08 / 2018$ & $100.000+$ & Gobierno & https://www.buenosaires.gob.ar/aplicacionesmoviles/ba-taxi-pasajeros \\
\hline BA Turismo & 4,3 & 355 & $08 / 08 / 2018$ & $50.000+$ & Gobierno & https://www.buenosaires.gob.ar/aplicacionesmoviles/ba-turismo \\
\hline Ciudad de Mendoza & 4,3 & 57 & $23 / 01 / 2018$ & $10.000+$ & Gobierno & https://ciudaddemendoza.gob.ar/turismo/ \\
\hline Cómo llego & 4 & 19.555 & $17 / 05 / 2018$ & $1.000 .000+$ & Gobierno & https://www.buenosaires.gob.ar/aplicacionesmoviles/ba-como-llego \\
\hline Denuncia Vial & 3 & 2.485 & $10 / 05 / 2018$ & $100.000+$ & Gobierno & https://www.buenosaires.gob.ar/aplicacionesmoviles/ba-denuncia-vial \\
\hline El mercado en tu barrio & 3,6 & 45 & $06 / 08 / 2018$ & $5.000+$ & Gobierno & https://www.argentina.gob.ar/elmercadoentubarrio \\
\hline Fronteras & 3,9 & 246 & $06 / 08 / 2018$ & $50.000+$ & Gobierno & $\mathrm{N} / \mathrm{D}$ \\
\hline La Muni con Vos & 3,1 & 122 & $09 / 08 / 2018$ & $1.000+$ & Gobierno & $\mathrm{N} / \mathrm{D}$ \\
\hline $\begin{array}{l}\text { Línea 144- Atención a } \\
\text { mujeres }\end{array}$ & 3,6 & 31 & $22 / 03 / 2018$ & $1.000+$ & Gobierno & https://www.argentina.gob.ar/linea144 \\
\hline Madhel & 4,6 & 37 & $12 / 07 / 2018$ & $1.000+$ & Gobierno & http://ais.anac.gob.ar/madhel/ \\
\hline Mi Anses & 3,9 & 15.319 & $24 / 04 / 2017$ & $1.000 .000+$ & Gobierno & https://www.anses.gob.ar/informacion/mi-anses \\
\hline Mi Argentina & 4,2 & 529 & $16 / 07 / 2018$ & $100.000+$ & Gobierno & https://www.argentina.gob.ar/miargentina \\
\hline Mi Monotributo & 4,4 & 6.087 & $13 / 09 / 2017$ & $100.000+$ & Gobierno & https://www.afip.gob.ar/celular/aplicaciones.asp \\
\hline Mi Próximo Colectivo & 2,4 & 31 & $10 / 11 / 2017$ & $1.000+$ & Gobierno & $\mathrm{N} / \mathrm{D}$ \\
\hline miBA & 3,2 & 148 & $20 / 07 / 2018$ & $10.000+$ & Gobierno & https://www.buenosaires.gob.ar/aplicacionesmoviles/miba \\
\hline $\begin{array}{l}\text { Monitor Integral SIO } \\
\text { Granos }\end{array}$ & 4,6 & 7 & $21 / 08 / 2018$ & $1.000+$ & Gobierno & $\mathrm{N} / \mathrm{D}$ \\
\hline $\begin{array}{l}\text { Museo Nacional del } \\
\text { Cabildo }\end{array}$ & 4,4 & 27 & $19 / 10 / 2016$ & $1.000+$ & Gobierno & $\begin{array}{r}\text { https://cabildonacional.cultura.gob.ar/noticia/conoce-la-aplicacion-del- } \\
\text { museo-del-cabildo/ }\end{array}$ \\
\hline Neuquén Guía de Viaje & 4,4 & 33 & $14 / 09 / 2015$ & $1.000+$ & Gobierno & $\mathrm{N} / \mathrm{D}$ \\
\hline Palpalá participa & 4,1 & 24 & $18 / 08 / 2016$ & $500+$ & Gobierno & http://www.palpala.gob.ar/index.php/palpar \\
\hline Precios en surtidor & 3,8 & 844 & $12 / 08 / 2017$ & $50.000+$ & Gobierno & https://www.minem.gob.ar/www/706/26165/precios-en-surtidor \\
\hline Radio Nacional Argentina & 4,4 & 351 & $07 / 02 / 2017$ & $10.000+$ & Gobierno & http://www.radionacional.com.ar/baja-la-app/ \\
\hline Seguridad Provincia & 4,3 & 876 & $02 / 07 / 2018$ & $100.000+$ & Gobierno & https://seguridad.gba.gob.ar/\#!/home \\
\hline $\begin{array}{l}\text { SIF Móvil Min. } \\
\text { Agroindustria }\end{array}$ & 4,6 & 23 & $26 / 04 / 2016$ & $1.000+$ & Gobierno & $\mathrm{N} / \mathrm{D}$ \\
\hline Tasas y Multas & 3,4 & 10 & $18 / 01 / 2016$ & $1.000+$ & Gobierno & $\mathrm{N} / \mathrm{D}$ \\
\hline Trenes en Directo & 3,6 & 8.670 & $12 / 03 / 2018$ & $500.000+$ & Gobierno & http://www.mininterior.gob.ar/tramitesyservicios/apps.php \\
\hline Turismo Santa Fe Ciudad & 4,7 & 11 & $03 / 09 / 2016$ & $1.000+$ & Gobierno & http://www.santafeturismo.gov.ar/ \\
\hline Ushuaia Turismo & 4,6 & 37 & $20 / 08 / 2017$ & $1.000+$ & Gobierno & $\mathrm{N} / \mathrm{D}$ \\
\hline
\end{tabular}

(1): N/D (No Disponible, en caso que no se encuentre enlace para descargar desde el sitio institucional). 


\subsection{Comentarios de Usuarios de Aplicaciones del Gobierno Argentino}

Con el objeto de realizar un análisis cuantitativo de los problemas identificados en los comentarios, en la Tabla 5 mostramos la relación de las 31 aplicaciones revisadas con las características definidas para la categorización de problemas. El tilde $(\checkmark)$ indica que para esa aplicación registramos, en los comentarios observados, al menos una evidencia de problemas relacionados con la característica asociada. Las celdas en blanco indican que no encontramos problemas sobre esa característica y para esa aplicación.

Tabla 5. Resumen de problemas identificados en los comentarios de usuarios.

\begin{tabular}{|c|c|c|c|c|c|c|c|c|c|c|c|c|c|}
\hline APLICACIONES & 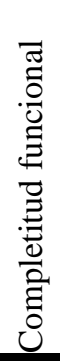 & 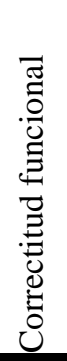 & 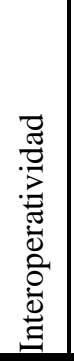 & 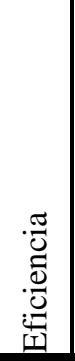 & 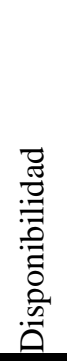 & 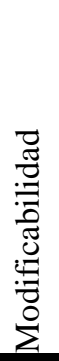 & 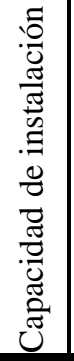 & 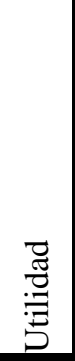 & 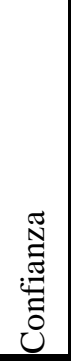 & 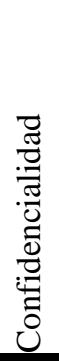 & 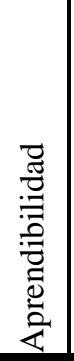 & 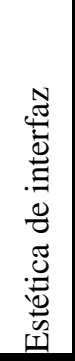 & 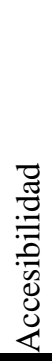 \\
\hline Línea 144- Atención a mujeres & $\checkmark$ & & & & $\checkmark$ & & & $\checkmark$ & $\checkmark$ & & & & \\
\hline Mi Argentina & $\checkmark$ & $\checkmark$ & & & & $\checkmark$ & $\checkmark$ & $\checkmark$ & $\checkmark$ & $\checkmark$ & $\checkmark$ & & \\
\hline Argentina Salud & & $\checkmark$ & $\checkmark$ & & & & & $\checkmark$ & $\checkmark$ & & & & \\
\hline El mercado en tu barrio & $\checkmark$ & $\checkmark$ & & & & & $\checkmark$ & $\checkmark$ & $\checkmark$ & & & & \\
\hline Fronteras & $\checkmark$ & $\checkmark$ & & $\checkmark$ & $\checkmark$ & & $\checkmark$ & $\checkmark$ & $\checkmark$ & & & & \\
\hline Precios en surtidor & $\checkmark$ & $\checkmark$ & & $\checkmark$ & $\checkmark$ & & $\checkmark$ & $\checkmark$ & $\checkmark$ & & $\checkmark$ & & \\
\hline Mi Monotributo & $\checkmark$ & $\checkmark$ & & $\checkmark$ & $\checkmark$ & & $\checkmark$ & & $\checkmark$ & & $\checkmark$ & & \\
\hline Mi Anses & & $\checkmark$ & & $\checkmark$ & $\checkmark$ & $\checkmark$ & & $\checkmark$ & . & & & & \\
\hline Seguridad Provincia & $\checkmark$ & $\checkmark$ & & & $\checkmark$ & & $\checkmark$ & $\checkmark$ & 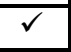 & & & & $\checkmark$ \\
\hline miBA & $\checkmark$ & $\checkmark$ & & $\checkmark$ & $\checkmark$ & & $\checkmark$ & $\checkmark$ & $\checkmark$ & & $\checkmark$ & & \\
\hline Denuncia Vial & $\checkmark$ & $\checkmark$ & & $\checkmark$ & & $\checkmark$ & & $\checkmark$ & $\checkmark$ & & $\checkmark$ & & \\
\hline Tasas y Multas & $\checkmark$ & $\checkmark$ & & $\checkmark$ & $\checkmark$ & & & & & & & & \\
\hline Palpalá participa & & $\checkmark$ & & $\checkmark$ & & & & $\checkmark$ & & & $\checkmark$ & & \\
\hline La Muni con Vos & $\checkmark$ & $\checkmark$ & & $\checkmark$ & $\checkmark$ & & $\checkmark$ & $\checkmark$ & 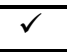 & & $\checkmark$ & & \\
\hline Trenes en Directo & $\checkmark$ & $\checkmark$ & & $\checkmark$ & $\checkmark$ & $\checkmark$ & & $\checkmark$ & $\checkmark$ & $\checkmark$ & $\checkmark$ & & \\
\hline Aerolíneas móvil & $\checkmark$ & $\checkmark$ & & $\checkmark$ & & & & $\checkmark$ & $\checkmark$ & & $\checkmark$ & $\checkmark$ & \\
\hline MADHEL & $\checkmark$ & & & $\checkmark$ & $\checkmark$ & & $\checkmark$ & & $\checkmark$ & & & & \\
\hline Mi Próximo Colectivo & $\checkmark$ & $\checkmark$ & $\checkmark$ & $\checkmark$ & $\checkmark$ & & $\checkmark$ & $\checkmark$ & $\checkmark$ & & & & \\
\hline BA Taxi & $\checkmark$ & & $\checkmark$ & & $\checkmark$ & $\checkmark$ & $\checkmark$ & $\checkmark$ & & & $\checkmark$ & & \\
\hline Cómo llego & $\checkmark$ & $\checkmark$ & & & $\checkmark$ & $\checkmark$ & & $\checkmark$ & $\checkmark$ & $\checkmark$ & & & \\
\hline BA Subtes & $\checkmark$ & $\checkmark$ & & $\checkmark$ & $\checkmark$ & $\checkmark$ & $\checkmark$ & $\checkmark$ & $\checkmark$ & $\checkmark$ & $\checkmark$ & $\checkmark$ & \\
\hline BA Turismo & $\checkmark$ & $\checkmark$ & & & & & $\checkmark$ & $\checkmark$ & & & $\checkmark$ & & \\
\hline Ciudad de Mendoza & $\checkmark$ & $\checkmark$ & & $\checkmark$ & $\checkmark$ & & & $\checkmark$ & $\checkmark$ & & $\checkmark$ & $\checkmark$ & \\
\hline Ushuaia Turismo & $\checkmark$ & & & & $\checkmark$ & & & $\checkmark$ & & & & & \\
\hline Turismo Santa Fe Ciudad & $\checkmark$ & & & & $\checkmark$ & & & & & & & & \\
\hline Neuquén Guía de Viaje & $\checkmark$ & & & $\checkmark$ & & & & & $\checkmark$ & & & & \\
\hline Alertamos & $\checkmark$ & & & $\checkmark$ & $\checkmark$ & $\checkmark$ & $\checkmark$ & $\checkmark$ & $\checkmark$ & & $\checkmark$ & $\checkmark$ & \\
\hline Museo Nacional del Cabildo & & & & $\checkmark$ & & & & & & & $\checkmark$ & $\checkmark$ & \\
\hline Radio Nacional Argentina & $\checkmark$ & & & $\checkmark$ & $\checkmark$ & $\checkmark$ & & $\checkmark$ & $\checkmark$ & & & & \\
\hline Monitor Integral SIO Granos & & & & & & & & & & & & & \\
\hline SIF Móvil Min. Agroindustria & $\checkmark$ & $\checkmark$ & & & & & & & & & $\checkmark$ & & \\
\hline $\begin{array}{ll}\text { TOTAL } \\
\end{array}$ & 26 & 21 & 3 & 19 & 20 & 9 & 14 & 23 & 21 & 4 & 16 & 5 & 1 \\
\hline
\end{tabular}


Estos datos muestran que la mayoría de las aplicaciones evidencian problemas relacionados con Completitud funcional. Esto ocurrió en 26 de las 31 aplicaciones estudiadas. Los usuarios manifiestan la necesidad de incluir nuevas funcionalidades, generalmente relacionadas con funcionalidad ofrecida por aplicaciones similares o por la versión web de la misma aplicación.

El siguiente grupo con más ocurrencias fue Utilidad, en 22 aplicaciones. En este caso, los comentarios indican que los usuarios no ven a la aplicación como un valor para ellos, o no perciben que la aplicación cumpla con sus necesidades. Otra característica con una importante cantidad de problemas (21 casos) fue Confianza: en esta, los usuarios reportan principalmente que la información que manejan las aplicaciones no está actualizada. Lo mismo ocurre con Correctitud funcional (21 casos), sobre la que se reporta que las aplicaciones no hacen lo que dicen que hacen. Por su parte, problemas de Disponibilidad se manifiestan en 20 casos, errores de instalación o funcionamiento que no permiten el uso de la aplicación. Y en 19 aplicaciones, se evidencias problemas de Eficiencia: aplicaciones muy lentas o que consumen muchos recursos de los dispositivos en los que se instala. El resto de las características registraron evidencia de problemas en menos del 50\% de las aplicaciones, siendo la característica con menor ocurrencia Accesibilidad, con 1 solo caso registrado. La Figura 1 grafica estos resultados.

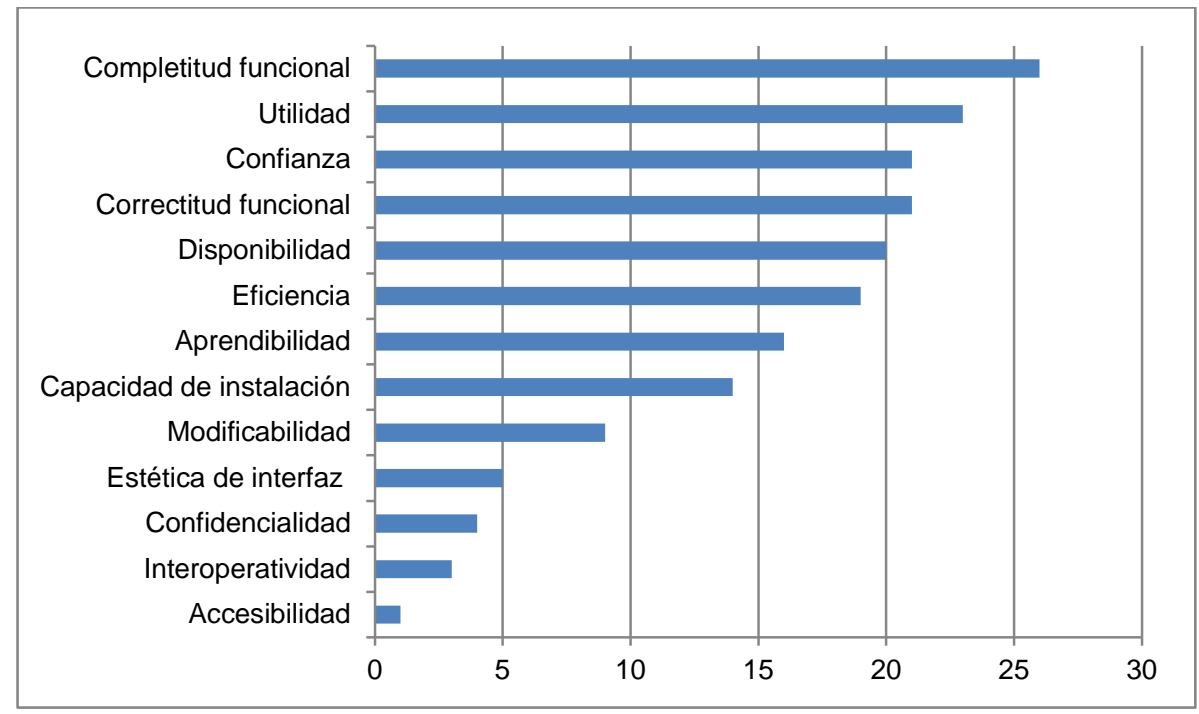

Figura 1. Cantidad de aplicaciones que evidencian problemas. (Fuente: Elaboración propia)

\subsection{Características de la Oferta de Aplicaciones del Gobierno Argentino}

Por otro lado, analizamos algunos aspectos relacionados con la oferta de las aplicaciones que podrían afectar la experiencia de los ciudadanos al interactuar con éstas.

Descargas. Solo 2 aplicaciones superan 1.000.000 de descargas: Mi ANSES y Cómo llego. El rango de mayor frecuencia de descarga es entre 1.000 y 5.000 veces, con 12 ocurrencias. Mientras que más de la mitad de las aplicaciones (17) fueron descargadas menos de 50.000 veces. Esto se muestra en la Figura 2. 


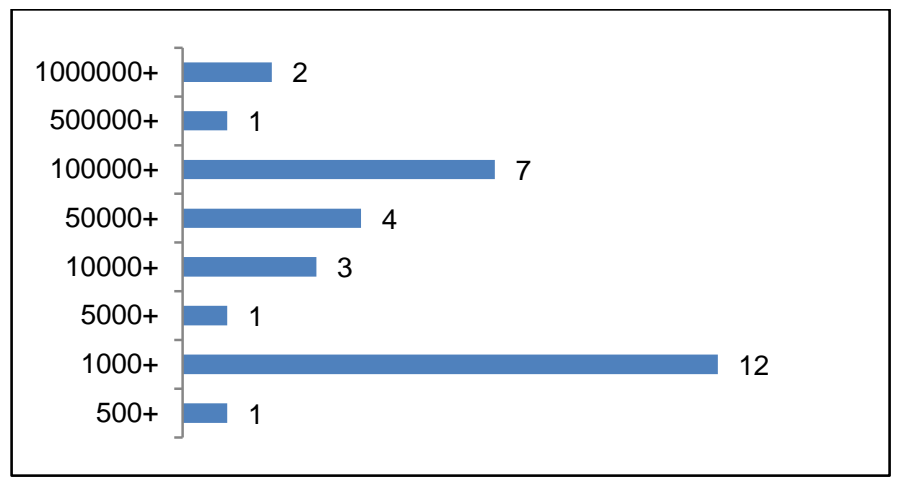

Figura 2. Frecuencia de descarga de aplicaciones móviles. (Fuente: Elaboración propia)

Valoración. El promedio de valoración de los usuarios de las aplicaciones es de 3,87 (en una escala de 1 a 5). Casi la mitad de las aplicaciones (45\%) registraron una valoración por debajo del valor promedio. Y solo el $10 \%$ tiene una valoración menor o igual a 3. Esto se ilustra en la Figura 3.

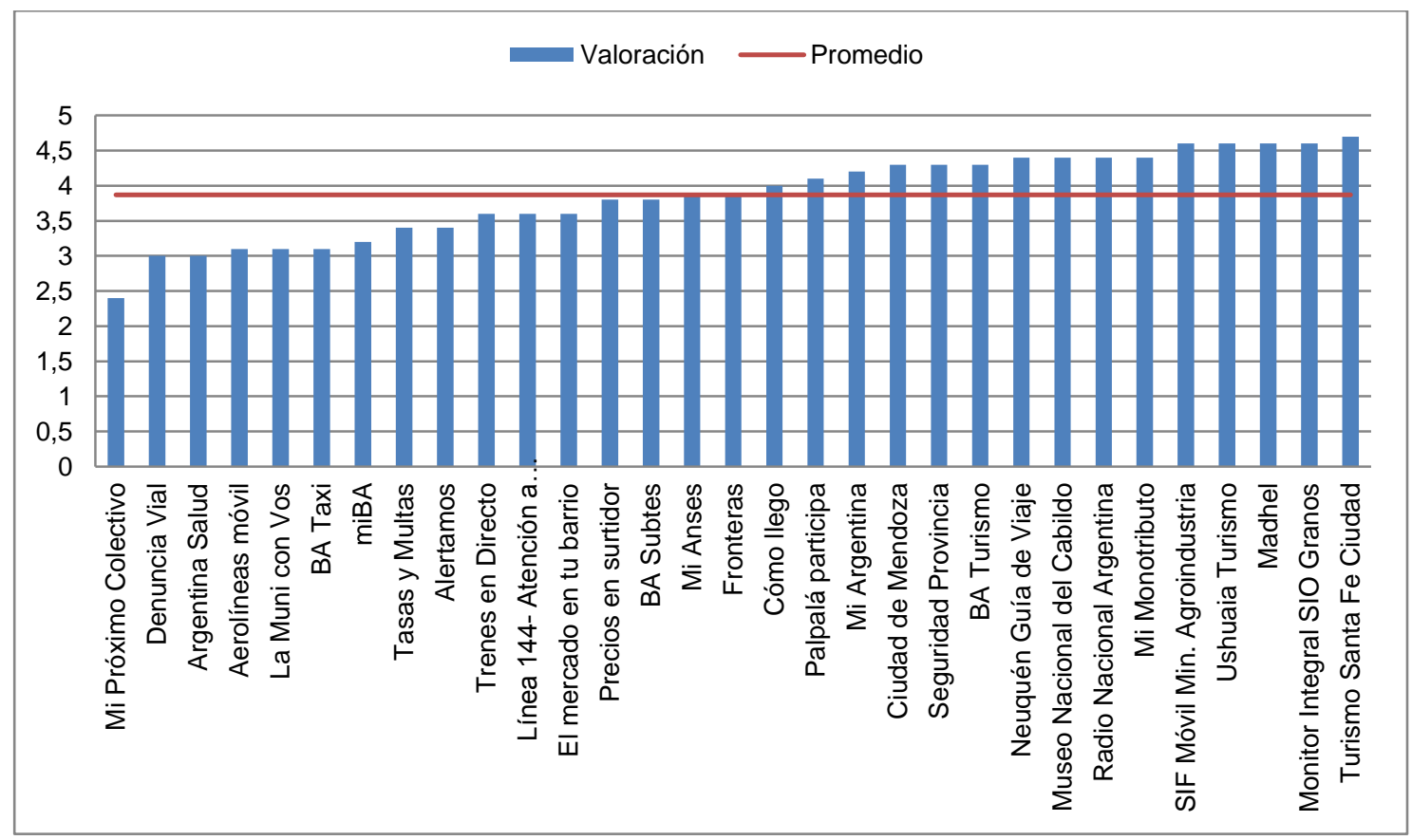

Figura 3. Promedio de valoración de aplicaciones móviles. (Fuente: Elaboración propia)

Sin embargo, la relación entre cantidad de opiniones y cantidad de descargas es muy baja. Como se observa en la 
Tabla 6, la aplicación que registra la valoración más alta (Turismo Santa Fe Ciudad), tiene 11 opiniones y más de 1.000 descargas. Esto significa que la calificación de 4,7 estrellas deriva de solo el 1,1\% de sus descargas. Mientras que la valoración más baja, que corresponde a la aplicación Mi Próximo Colectivo (2,4 estrellas), se basa en las opiniones del 3,1\% de los usuarios que descargaron la aplicación. 
Tabla 6. Relación entre cantidad de opiniones y cantidad de descargas. (Fuente: Elaboración propia)

\begin{tabular}{|l|r|r|r|r|}
\hline Aplicación & Descargas & Opiniones & $\begin{array}{r}\text { \% Usuarios } \\
\text { que opinan }\end{array}$ & Valoración \\
\hline Turismo Santa Fe Ciudad & 1.000 & 11 & $1,1 \%$ & 4,7 \\
\hline Monitor Integral SIO Granos & 1.000 & 7 & $0,7 \%$ & 4,6 \\
\hline SIF Móvil Min. Agroindustria & 1.000 & 23 & $2,3 \%$ & 4,6 \\
\hline Madhel & 1.000 & 37 & $3,7 \%$ & 4,6 \\
\hline Ushuaia Turismo & 1.000 & 37 & $3,7 \%$ & 4,6 \\
\hline Museo Nacional del Cabildo & 1.000 & 27 & $2,7 \%$ & 4,4 \\
\hline Neuquén Guía de Viaje & 1.000 & 33 & $3,3 \%$ & 4,4 \\
\hline Radio Nacional Argentina & 10.000 & 351 & $3,5 \%$ & 4,4 \\
\hline Mi Monotributo & 100.000 & 6087 & $6,1 \%$ & 4,4 \\
\hline Ciudad de Mendoza & 10.000 & 57 & $0,6 \%$ & 4,3 \\
\hline BA Turismo & 50.000 & 355 & $0,7 \%$ & 4,3 \\
\hline Seguridad Provincia & 100.000 & 876 & $0,9 \%$ & 4,3 \\
\hline Mi Argentina & 100.000 & 529 & $0,5 \%$ & 4,2 \\
\hline Palpalá participa & 500 & 24 & $4,8 \%$ & 4,1 \\
\hline Cómo llego & 1.000 .000 & 19555 & $2,0 \%$ & 4 \\
\hline Mi Anses & 1.000 .000 & 15319 & $1,5 \%$ & 3,9 \\
\hline Fronteras & 10.000 & 246 & $2,5 \%$ & 3,9 \\
\hline Precios en surtidor & 50.000 & 844 & $1,7 \%$ & 3,8 \\
\hline BA Subtes & 100.000 & 2763 & $2,8 \%$ & 3,8 \\
\hline El mercado en tu barrio & 5.000 & 45 & $0,9 \%$ & 3,6 \\
\hline Trenes en Directo & 500.000 & 8670 & $1,7 \%$ & 3,6 \\
\hline Línea 144- Atención a mujeres & 1.000 & 31 & $3,1 \%$ & 3,6 \\
\hline Alertamos & 50.000 & 399 & $0,8 \%$ & 3,4 \\
\hline Tasas y Multas & 1.000 & 10 & $1,0 \%$ & 3,4 \\
\hline miBA & 10.000 & 148 & $1,5 \%$ & 3,2 \\
\hline BA Taxi & 100.000 & 554 & $0,6 \%$ & 3,1 \\
\hline Aerolíneas móvil & 100.000 & 2448 & $2,4 \%$ & 3,1 \\
\hline La Muni con Vos & 1.000 & 122 & $12,2 \%$ & 3,1 \\
\hline Argentina Salud & 1.000 & 10 & $1,0 \%$ & 3 \\
\hline Denuncia Vial & 100.000 & 2485 & $2,5 \%$ & 3 \\
\hline Mi Próximo Colectivo & 1.000 & 31 & $3,1 \%$ & 2,4 \\
\hline
\end{tabular}

Promoción. La promoción del uso de las aplicaciones desde sitios web institucionales se manifiesta por medio de enlaces a la tienda de aplicaciones en $71 \%$ de los casos. Solo en 9 de los sitios web oficiales no fue posible encontrar un enlace que direccione hacia las tiendas de aplicaciones.

Actualización. Más de la mitad de las aplicaciones (55\%) habían sido actualizadas dentro de los 6 meses anteriores a la realización de este estudio; mientras que 35\% de ellas no lo hacían desde hacía más de 1 año. La Tabla 7 resume el cálculo de la cantidad de días transcurridos desde la última actualización, ordenados en rangos de 3 meses. 
Tabla 7. Tiempo transcurrido desde la última actualización. (Fuente: Elaboración propia)

\begin{tabular}{|l|r|r|r|}
\hline Rango & Frecuencia & \multicolumn{1}{|c|}{ Porcentaje } & Porcentaje acumulado \\
\hline Menos de 3 meses & 11 & 35 & 35 \\
Entre 3 y 6 meses & 6 & 19 & 55 \\
Entre 6 y 9 meses & 1 & 3 & 58 \\
Entre 9 y 12 meses & 2 & 6 & 65 \\
Más de 1 año & 11 & 35 & 100 \\
\hline
\end{tabular}

Mantenimiento. La responsabilidad de mantener las aplicaciones actualizadas en la tienda de aplicaciones es del gobierno, ya que en $95 \%$ de las aplicaciones, el gobierno es titular de las cuentas en la tienda. Solo $5 \%$ son administradas por entidades externas.

\section{CONCLUSIONES}

En este estudio exploramos información disponible en la web para identificar posibles problemas asociados a la experiencia de usuarios con el gobierno móvil en Argentina.

El análisis de los comentarios de la tienda de aplicaciones reveló que los usuarios demandan aplicaciones de gobierno enfocadas en servicios útiles y beneficios tangibles para los ciudadanos, y también reclaman aplicaciones que brinden información actualizada y que estén disponibles en cualquier momento.

Por otro lado, el análisis de la oferta de aplicaciones de gobierno en Argentina, mostró que la cantidad de descargas es reducida, que las aplicaciones no son actualizadas con frecuencia, y que la promoción de su uso desde sitios web y redes sociales institucionales es limitada. En relación a esto, también se detectó la necesidad de mantener actualizada la oferta publicada en el sitio de aplicaciones móviles del Estado argentino, eliminando del listado aquellas aplicaciones que ya no están disponibles en la tienda, y tal vez incorporando otras aplicaciones que aún no figuran en este sitio.

Si bien el concepto de gobierno móvil promueve el desarrollo de servicios eficientes para satisfacer las necesidades de distintos usuarios, los resultados de este estudio identificaron deficiencias que afectan negativamente la experiencia de los ciudadanos al interactuar con las aplicaciones móviles del Estado argentino.

Finalmente, el estudio realizado generó información potencialmente útil para mejorar estas aplicaciones de gobierno móvil. Sin embargo, no podemos asegurar que todos los problemas de experiencia de usuario identificados son problemas efectivos. Por lo tanto, en trabajos futuros, pretendemos complementar este estudio aplicando otras técnicas de evaluación que permitan no solo verificar los resultados obtenidos en este estudio sino también analizar otros aspectos importantes de la UX.

\section{AGRADECIMIENTOS}

A la UNPA por el soporte al Proyecto de Investigación 29/B222: "Diseño y Evaluación de Experiencia de Usuario para Multi-Dispositivos". 


\section{REFERENCIAS}

AL-NUAIM, H. (2014). Context-Aware Mobile Interface Design for M-government. UserCentric Technology Design for Nonprofit and Civic Engagements, Public Administration and Information Technology. Springer International Publishing.

BALLARD, B. (2007). Designing the Mobile User Experience. John Wiley \& Sons.

CARRIZO, A., GAETÁN, G., SALDAÑO, V., \& MARTIN, A. (2018). Análisis de la Experiencia de Usuario con Aplicaciones de Gobierno Móvil en Argentina. Libro de Resúmenes del V Encuentro de Investigadores, Becarios y Tesistas de la Patagonia Austral, (pp. 483-487). Rio Gallegos.

CLAD. (2007). Carta Iberoamericana de Gobierno Electrónico. IX Conferencia Iberoamericana de Ministros de Administración Publica y Reforma del Estado. Pucón, Chile: Centro Latinoamericano de Administración para el Desarrollo.

COSTOPOUlOU, C., \& MOLHANEC, M. (2014). Evolution Towards Mobile Government: The Greek and the Czech Cases. Springer International Publishing.

GALVIS CARREÑO, L. V., \& WINBLADH, K. (2013). Analysis of user comments: an approach for software requirements evolution. Proceedings of the 2013 International Conference on Software Engineering (pp. 582-591). IEEE Press.

HALL, E. (2013). Just enough research. New York: A Book Apart.

HARTSON, R., \& PYLA, P. S. (2012). The UX Book: Process and Guidelines for Ensuring a Quality User Experience. Morgan Kaufmann. Elsevier.

HEWSON, C. M., \& LAURENT, D. (2017). Research design and tools for Internet research. In N. Fielding, R. Lee, \& G. Blank (Eds.), The Sage Handbook of Online Research Methods (2nd ed.). SAGE Publications Ltd.

IACOB, C., \& HARRISON, R. (2013). Retrieving and analyzing mobile apps feature requests from online reviews. Proceedings of the 10th Working Conference on Mining Software Repositories (pp. 41-44). IEEE Press.

INSTITUTO NACIONAL DE ESTADÍSTICA Y CENSOS. (2019). Acceso y uso de tecnologías de la información y la comunicación. EPH. Cuarto trimestre de 2018. Buenos Aires: Publicaciones del INDEC.

ISO/IEC JTC 1/SC 7. (2011). ISO/IEC 25010:2011. Software engineering - Software product Quality Requirements and Evaluation (SQuaRE) - System and software quality models. International Organization for Standardization.

ISO/TC 159/SC 4. (2018). ISO 9241-11:2018. Ergonomics of human-system interaction -Part 11: Usability: Definitions and concepts. International Organization for Standardization. 
LÖNN, C. M. (2014). An m-government solution for complaint and problem management: Designing a solution for government 2.0. Department of Computer and Systems Sciences, Stockholm University.

NASER, A., \& CONCHA, G. (2011). El gobierno electrónico en la gestión pública. Santiago de Chile: Naciones Unidas.

NIELSEN, J. (1995). 10 usability heuristics for user interface design. Retrieved from Nielsen Norman Group: www.nngroup.com/articles/ten-usability-heuristics/

NIELSEN, J. (1999). Designing Web Usability. New Riders.

NIELSEN, J., \& BUDIU, R. (2015). User Experience for Mobile Applications and Websites. Design Guidelines for Improving the Usability of Mobile Sites and Apps (3rd ed.). California: Nielsen Norman Group.

NORMAN, D. (2013). The design of everyday things: Revised and expanded edition. Basic Books.

NTALIANI, M., COSTOPOULOU, C., \& KARETSOS, S. (2008). Mobile government: A challenge for agriculture. Government Information Quarterly , 25 (4), 699-716.

OECD/International Telecommunication Union . (2011). M-Government: Mobile Technologies for Responsive Governments and Connected Societies. Paris: OECD Publishing.

ROBERTSON, S. P., \& VATRAPU, R. K. (2010). Digital government. Annual Review of Information Science and Technology, 44 (1), 317-364.

RONAGHAN, S. A. (2002). Benchmarking E-government, a Global Perspective: Assessing the UN Member States. New York: United Nations Division for Public Economics and Public Administration.

TRAVIS, D. (2009). 247 web usability guidelines. Retrieved from Userfocus: www.userfocus.co.uk/resources/guidelines.html

W3C-WAI. (n.d.). Introduction to Web Accessibility. (L. H. Shawn, Ed.) Retrieved from World Wide Web Consortium (W3C) Web Accessibility Initiative (WAI): https://www.w3.org/WAI/fundamentals/accessibility-intro/

YU, C. C. (2014). Developing Value-Centric Business Models for Mobile. Conference on Electronic Government (EGOV) (pp. 325 -336). Dublin, Ireland: IFIP International Federation for Information Processing.

ZAMZAMI, I., MAHMUD, M., \& ABUBAKAR, A. (2014). Information Quality Evaluation of mobile-Government (mGovernment) Services. HCI International 2014 - Posters' Extended Abstracts (pp. 280-285). Springer. 


\section{ANEXO I. Categorización de Comentarios de Usuarios.}

Datos recolectados de los cometarios de usuarios sobre las aplicaciones de gobierno móvil en Argentina. Por cada aplicación, se muestra un ejemplo de comentario asociado a las categorías de problema identificadas.

\section{Línea 144- Atención a mujeres.}

Completitud Funcional Mi novia sufrió una relación violenta. La probó y realmente no siente que sirva. Un novio que revise celular y vea esa app no creo que lo tome bien. No tiene botón antipánico, no se puede ocultar de ninguna forma, podría envíar notificaciones push camufladas x ej. Deberían rediseñarla juntándose con especialistas de centros contra la violencia de género para poder darle verdadera utilidad.

\begin{tabular}{ll}
\hline Disponibilidad & Mandé un mail y ni respuesta, ya van 2 semanas \\
\hline Utilidad & $\begin{array}{l}\text { No sirve!!! Y encima llamas al } 144 \text { y tampoco atiende nadie!!!! Estuve 20min. Esperando y na- } \\
\text { die contestó }\end{array}$ \\
\hline Confianza & $\begin{array}{l}\text { Faltan cosas, acceso a ubicación apenas instalo la aplicación y de ahí un botón de pánico, } \\
\text { por que la encuesta no siempre puede ser respondida a conciencia y además la opción de } \\
\text { elección de género, las preguntas están echas para la mujer y no siempre es la víctima. Gra- } \\
\text { cias }\end{array}$ \\
\hline
\end{tabular}

\section{Mi Argentina.}

Completitud Funcional
Todavía no funciona al 100\% pero cumple la función de mostrar el registro de conducir. Seria bueno añadir toda documentación inportante: Seguro, Dni (pero que sirva para mostrarlo, actualmente no tiene validez legal), Tarjeta SUBE, obra social, etc. Y realizar trámites como pagar arba, chequear mult.

Correctitud Funcional pesima. no te muestra el DNI y la licencia de conducir virtual nunca la suben.. una mentira mas de este gobierno para quedarse con nuestros datos y aceptemos en las bases y condiciones que nos espien empresas como google y demas

\begin{tabular}{ll}
\hline Modificabilidad & $\begin{array}{l}\text { Instale la aplicación en mi celular y anda perfecto, pero al querer actualizarla me dice que ya } \\
\text { no es compatible con mi teléfrono Xiaomi Redmi Note } 4 \text { con android 7, les envié varios mail } \\
\text { por este tema y consultas por este medio, porqué no me responden??, o no saben qué res- } \\
\text { ponder?? }\end{array}$ \\
\hline Capacidad de instalación & $\begin{array}{l}\text { A los desarrolladores: Tengo un Samsun S7 y al querer instalar la APP me indica que mi dis- } \\
\text { positivo no es compatible. Por que? Gracias por su ayuda. }\end{array}$ \\
\hline Utilidad & NO SIRVEN LOS TURNOS QUE SACAS!! ES TRUCHO \\
\hline Confianza & $\begin{array}{l}\text { validas tú identidad pero la aplicación establece una dirección de hace más de 10 años en } \\
\text { donde no vivís y que ni siquiera está en tú dni. }\end{array}$ \\
\hline Confidencialidad & $\begin{array}{l}\text { Esta buena la idea pero pondría una constraseña antes de iniciar la app por robos o perdi- } \\
\text { das }\end{array}$ \\
\hline Aprendibilidad & $\begin{array}{l}\text { Malisima la app. Primero, una interfaz poco amigable e intuitiva. Te perdes entre todas las } \\
\text { opciones. Segundo, el proceso de validacion no lo logre relaizar, mas de 20 intentos y nada. }\end{array}$ \\
\hline
\end{tabular}

\section{Argentina Salud.}

Correctitud Funcional eata bien pero en Miramar donde vivo no fuguran ninguno de los CAPS solo el hospital y una clinica privada 


\begin{tabular}{|c|c|}
\hline Interoperabilidad & $\begin{array}{l}\text { Es una vergüenza para todos los argentinos tener una aplicación tan precaria como ésta no } \\
\text { Integra a la agenda del celular, no permite llevar la planificación de las vacunas, no permite } \\
\text { llevar una historia clínica de la familia, es una porquería por favor no instale esta aplicación. } \\
\text { No entiendo como... }\end{array}$ \\
\hline Utilidad & $\begin{array}{l}\text { Pocas funciones útiles, no sirve para futuros historiales médicos en la aplicación, es muy } \\
\text { básica y no esta optimizada, y no hacen que las instituciones estén inscriptas digitalmente, } \\
\text { no están formalizadas. }\end{array}$ \\
\hline Confianza & $\begin{array}{l}\text { Es completamente inútil, en toda la Provincia de Buenos Aires solo me arroja } 3 \text { centros de } \\
\text { salud donde donar sangre y los } 3 \text { privados. Papelón. }\end{array}$ \\
\hline
\end{tabular}

El Mercado en tu Barrio.

Completitud Funcional Esta bien solo falta lo más importante, los precios del momento en cada feria.

\begin{tabular}{ll}
\hline Correctitud Funcional & $\begin{array}{l}\text { Tiene errores de ubicación. Vivo a } 3 \mathrm{~km} \text {. de la feria de Morón y la aplicación señala } 5.200 \\
\mathrm{~km} \text {. Y no es la única. }\end{array}$ \\
\hline Capacidad de Instalación & Dice q no se pude instalar da error \\
\hline Utilidad & Es toda una mentira.NO vayan pierden tiempo y dinero.NO existe NINGUNA feria \\
\hline Confianza & $\begin{array}{l}\text { Es una verguenza no tiene filtros de busqueda y en florencio varela no figura ni una feria ni } \\
\text { as hay. Tampoco existieron nunca los precios cuidados.el estado es muy iresponsable son } \\
\text { sura propaganda aprovecho para decir que la plaza es un desastre todo roto. }\end{array}$
\end{tabular}

\section{Fronteras.}

Completitud Funcional

\begin{tabular}{ll} 
& $\begin{array}{l}\text { vamente). 2- suponiendo que siempre hay buena comunicacion con personal del otro lado, } \\
\text { se podría informar con calendarios o de algun modo las fechas de funcionalidad del puerto } \\
\text { de ambos lados. eso estaría muy bien y sería util. gra }\end{array}$ \\
\hline Correctitud Funcional & $\begin{array}{l}\text { Completamente inutil Una pérdida de tiempo y plata de todos los argentinos hay colas de } \\
10 \mathrm{~km} \text { en paso a chile por mendoza y la aplicación dice tránsito normal. Una burla. Una por } \\
\text { quería la aplicación. }\end{array}$ \\
\hline Eficiencia & $\begin{array}{l}\text { Bastante util. Pero en ciertos momentos la app no devuelve resultados, como también no } \\
\text { actualizan constantemente }\end{array}$ \\
\hline Capacidad de Instalación & No arranca. No pasa nada \\
\hline Utilidad & $\begin{array}{l}\text { No sirve No está actualizado a la hora, en pasos de montaña no indica tipo de vehículos ni } \\
\text { tipo de camino no todos tienen 4x4, toco en icono en el mapa y sale cualquier verdura, se- } \\
\text { ría una buena herramienta siempre y cuando tenga actualizaciones más frecuentes quizá } \\
\text { cada } 15 \text { min pero no cada } 6 \text { horas }\end{array}$ \\
\hline Confianza & $\begin{array}{l}\text { Debería figurar fecha y hora de la información, para evitar tener información desactualiza- } \\
\text { da.. } 0 \text { al menos saberlo. }\end{array}$ \\
\hline
\end{tabular}

excelente aplicación!!! con algunos detalles como: 1- la información es escasa, cuando esta cerrado el paso de vehículos, se debería informar que está cerrado por tal motivo (de esa forma no entramos en la duda de que hay paso pero esta bloqueado o no hay paso definitivamente). 2- suponiendo que siempre hay buena comunicacion con personal del otro lado, se podría informar con calendarios o de algun modo las fechas de funcionalidad del puerto
Precios en Surtidor.

Completitud Funcional

\begin{tabular}{ll}
\hline Correctitud Funcional & $\begin{array}{l}\text { es mentira ...fui a una estación d servicio q supuestamente estaba mas barato el combusti. } \\
\text { ble y nada q ver }\end{array}$ \\
\hline Eficiencia & Resulta muy lenta (casi imposible) la aplicación para moverse entre provincias \\
\hline Disponibilidad & no siempre se localiza automáticamente donde uno se encuentra \\
\hline
\end{tabular}




\begin{tabular}{ll}
\hline Capacidad de Instalación & A5 No me funciona \\
\hline Utilidad & - No carga correctamente los mapas. - Faltan estaciones, al menos de GNC, ej Rosario de la \\
& Frontera, Metan, Tilcara... \\
\hline Confianza & $\begin{array}{l}\text { los precios no están actualizados o debidamente verificados, me pasó de ir a } 2 \text { estaciones } \\
\text { en que el precio de surtidor no era el mismo que decía la app }\end{array}$ \\
\hline Aprendibilidad & $\begin{array}{l}\text { El modo lista no permite presionar sobre la estación y que te la muestre en el mapa, creo } \\
\text { que esto sería fundamental. }\end{array}$ \\
\hline
\end{tabular}

Mi Monotributo.

Completitud Funcional muy práctica, podria permitir la facturación desde la misma app

\begin{tabular}{ll}
\hline Correctitud Funcional & Falta poder ver y descargar el comprobante de pago \\
\hline Eficiencia & muchos problemas pra conectar . \\
\hline Disponibilidad & genial, solo q a veces no puedo pagar online \\
\hline Capacidad de Instalación & $\begin{array}{l}\text { Pésima app. No me dejo ingresar porque informa que la app no funciona en dispositivos } \\
\text { rooteados, cuando mi s8 es nuevo y obviamente no esta modificado ni nada por el estilo. } \\
\end{array}$ \\
\hline Malisimos!! Perdida de tiempo. No la instalen. \\
\hline Una aplicación con información tan sensible, no puede tener 1.5 años sin actualizar, esta- \\
mos en 2019 señores \\
\hline
\end{tabular}

\section{Mi Anses.}

\begin{tabular}{ll} 
Correctitud Funcional & $\begin{array}{l}\text { muy mala no se puede entrar a ninguna de la opciones, espero que lo mejoren rapido por- } \\
\text { que esta apps seria de mucha utilidad para varias personas. }\end{array}$ \\
\hline Eficiencia & PESIMO NO ANDA QUEDA TILDADO \\
\hline Disponiibilidad & una porquería. van como 3 veces q la instalo y desinstalo y aún así no me deja entrar. dice q \\
& no estoy autorizado para entrar en esta página. desde la web entró sin problemas!solucio- \\
& nen el problema urgente!! \\
\hline Modificabilidad & $\begin{array}{l}\text { No me gusta la nueva actualización por que no puedo ver mis turnos.. antes si podia hacer- } \\
\text { lo...es muy importante poder verlos... cuando ingreso en mis turnos y entró a mi cuenta se va } \\
\text { a consultas y cuando quiero ver mis turnos me dice cerrar cesión }\end{array}$ \\
\hline muy mala...m piden nro d cuil les doy m piden nro d clave social la cual no tengo ...pogo \\
continuar y no puedo xq no tengo la clave...m obligan a realizarla...la voy y la hago.....y con- \\
testo preguntas y no m la da xq hay un error...error d q?... solo queria el pin d la sube para \\
avegiguar mi saldo....y m la complicaron....xq la clave t la dan para bajar un formulario y pre- \\
sentar nosr en dond....xq la complican?
\end{tabular}

\section{Seguridad Provincia.}

Completitud Funcional falta boton antipánico. las denuncias no son anónimas, desde que la app obtiene datos del movil. nadie se animará a denunciar a funcionarios públicos.

\begin{tabular}{ll}
\hline Correctitud Funcional & $\begin{array}{l}\text { Mala... Conoce tu comisario no tiene datos. Dependencias policiales no tiene datos de comi- } \\
\text { sarias ni números telefónicos, solo destacamentos. }\end{array}$ \\
\hline Disponibilidad & $\begin{array}{l}\text { No sirve.abro la app y solo me aparece toda la pantalla en blanco e tampoco la puedo des- } \\
\text { cargar de mi tableta }\end{array}$ \\
\hline Capacidad de Instalación & $\begin{array}{l}\text { La instale y desinstale } 3 \text { veces y jamas la pude abrir a pesar de haber probado } 2 \text { teléfonos } \\
\text { diferentes. Le doy cero punto }\end{array}$ \\
\hline
\end{tabular}




\begin{tabular}{ll}
\hline Utilidad & No sirve. Hice la denuncia en la aplicacion, aportando todos mis datos personales, mencio- \\
nando que en el barrio acontecieron una serie de robos en horario matutino por delicuentes \\
que se desplazan en moto robandole a los vecinos. Paso siguiente me dice la app que debo \\
"ratificar la denuncia en la fiscalia" para que se tome en serio!! jaja Señores funcionarios en- \\
tonces para que inventaron esta PORQUERIA? No tenemos tiempo, ocupamos gran parte de \\
nuestro dia trabajando, ustedes solo venden humo! \\
\hline Confidencialidad & Engañosa. Me dice que no hay ninguna denuncia hecha. Inoperantes \\
\hline Accesibilidad & Nose puede denunciar soy sordo hipoacusico \\
\hline
\end{tabular}

miBA.

Completitud Funcional Tienen que incluir todos los servicios de la página, no lo que les conviene por publicidad

Correctitud Funcional vo sirve para solicitar la tarjeta MiBA para bicis (y el formulario web tampoco).

Eficiencia Lenta ineficiente y da puros errores a las consultas Si pagaron por está aplicación q les de-
vuelvan la plata q es plata de ntros impuestos

Disponibilidad No me deja ni cambiar los datos de mi perfil así ni puedo usarla debidamente.

\section{Capacidad de Instalación} No me deja ni loguearme ni crear un nuevo usuario. sale un código de error! solucionenlo porque necesito utilizar la app

Utilidad poco útil. la información que tiene es irrelevante. para colmo ni siquiera tiene información sobre los turnos, como para darle algo de utilidad.

Confianza

Para Ecobici instalar "BA Ecobici por Tembici" no esta. Ecobici: publica información falsa, de 6 estaciones visitadas hoy con disponibilidad en la app, todas fuera de servicio o apagadas y sin bicicletas (Hoy iniciaron cambio de sistema). No hay informacion de la novedad. El GPS no actualiza información en el mapa de bicis, y los mapas no permiten consultar mi ubicación actual. Tampoco el trayecto de mi supuesta ubicación a la estación. Completamente inútil. Ciudad hoy: noticia de hace 5 meses

\begin{tabular}{l} 
Aprendibilidad \\
\hline Denuncia Vial. \\
Completitud Funcional
\end{tabular}

Dificil utilizar

\section{Completitud Funcional}

No indica la resolución del caso, simplemente informa que se acepta el caso e indica que se tomarán las medidas correspondientes. Que medidas? Estoy subiendo fotos al divino botón? Me podrían indicar si se efectuó la multa? Cuando? Estimo que si uno se toma el trabajo de cooperar, sería bueno que tenga una devolución que lo estimule a seguir haciéndolo. Independientemente de eso, la aplicación me resultó muy práctica. No me tomó una segunda infracción de mismo vehículo, misma locación, dist mes.

Correctitud Funcional malísimo baje la app para denunciar a los autos que se estacionan en mi garaje y cuando lo quiero abrir te piden Número de DNI y todos mis datos elegidos la opcion entrar por Facebook, cuando lo hago vuelve a la planilla de rellenar con mis datos, NUNCA PUDE HACER LA DENUNCIA, USAN ESTOS MEDIOS PARA PONERTE EN SUS LISTAS COMO PARTIDARIOS DE SU POLITICAS, SOY ANTIPoliticos descreo de todos ellos

\begin{tabular}{ll}
\hline Eficiencia & se cuelga todo el tiempo. \\
\hline Modificabilidad & Cambie de equipo y ya no me muestra el historial de denuncias realizadas. Arreglen eso! \\
\hline Utilidad & $\begin{array}{l}\text { Las infracciones no impactan. Hice la consulta y ninguno de los que estaciona en mi garage } \\
\text { sin dejarme acceder tiene la multa. Desastre. Fracaso total. Mucha tecnologías al pedo por- } \\
\text { que no sirven de nada. }\end{array}$ \\
\hline Confianza & Está bueno el sistema, pero hace 2semanas que no me muestran las denuncias que hice. y \\
& nose cómo fueron resueltas. Estaría bueno que me solucionen ese inconveniente. \\
\hline Aprendibilidad & como se usa? pongo registrar la cuenta con face, pongo todos mis datos y vuelvo a la pan- \\
& talla inicial.... \\
\hline
\end{tabular}


Tasas y Multas.

Completitud Funcional

No tiene para cargar la patente de Mercosur, 2018, es 1 vergüenza

Correctitud Funcional

Malísima Pones el dni y te dice error pones la patente y también una porquería

Eficiencia

Horrible. Hibrida. Lenta. Hace más de un año que existe la patente Mercosur y solamente admite las anteriores

Disponibilidad

No sirve da error todo el tiempo Malisimaaaaa

Palpalá Participa.

Correctitud Funcional No deja adjuntar fotos solo la queja, ahora a esperar que por lo menos 1 queja sea respondida

\begin{tabular}{ll}
\hline Eficiencia & Se cierra solo \\
\hline Utilidad & $\begin{array}{l}\text { No puedo hacer reclamo No puedo hacer reclamo. Parece que como no subo la foto, no me } \\
\text { deja grabar el reclamo. PROBLEMA SOLUCIONADO! Gracias! }\end{array}$ \\
\hline Aprendibilidad & Muy buena iniciativa Falta un video como usarlo.. después esta bien. \\
\hline
\end{tabular}

La Muni con Vos.

Completitud Funcional

de una imagen > Permitir "Otro" en "Tipo de reporte". No siempre la situación se ajusta al listado de opciones. > Agregar una funcionalidad de "Comentarios" que sea de texto libre: Leo gente acá en los comentarios agradeciendo o reclamando al intendente y no tiene nada que ver con la app. > Permitir comentarios sobre un reporte existente: Para hacer un seguimiento del incidente o agregar información. Finalmente, y esto corresponde a la web: Cuando comparto un incidente mío, el link tiene el ID de reporte, pero al entrar a la web se pierde el ID

Correcitud Funcional La idea está buena. Lástima q no se pueden cargar reportes. No te deja seleccionar catego. ría.....y doy fe que en un principio funcionaba....que pasó?

Eficiencia No carga las denuncias es muy lent a y no podes cargar mas de 4 fotos.y no atienden los re-
clamos.estariá bueno q lo solucionen. sería muy util

Disponibilidad no funciona en ningún teléfono, LGQ6, galaxy \$7, moto g6, en ninguno inicia la aplicación, ni eso hacen bien, desarrolladores de aplicaciones inservibles contrataron

Capacidad de Instalación no funciona en ningún teléfono, LGQ6, galaxy $\$ 7$, moto g6, en ninguno inicia la aplicación, ni eso hacen bien, desarrolladores de aplicaciones inservibles contrataron

Utilidad La idea me pareció excelente, sin embargo no puedo completar el formulario debido a que categoría y tipo de reporte no se despliegan.para seleccionar una opción. En definitiva no puedo enviar ningún reporte. Por resuelva lo rápido, de lo contrario solo será inútil. Gracias

Confianza Ingresas y ves miles de reportes sin resolver de hace tiempo... Ojalá mejore, cuándo hago la gestión telefónica lo solucionan dentro de las 72 hs mínimo.

Aprendibilidad Anda bien en un SIII El único inconveniente que le veo es que para hacer un reclamo de otro rubro tengo que volver a ubicar el mapa en donde estoy. Se podría fijar por defecto en el último lugar reclamado y que se permita mover desde alli si se quiere cambiar el lugar. Por lo demás registro y envío andan muy bien.

\section{Trenes en Directo.}

Completitud Funcional deberia mandar notificación cuando un servicio no funciona

Correctitud Funcional no sirve; sólo informa horarios programados y no los reales. Hay media hora de atraso hasta el momento, yo acá sigo esperando el tren y la app dice que ya pasó a horario y el estado es "NORMAL"

Eficiencia la app se queda funcionando en segundo plano y consume batería. tengo que forzar el cierre. 


\begin{tabular}{ll}
\hline Disponibilidad & $\begin{array}{l}\text { lamentablemente se cuelga la instalé en mi dispositivo y me sale una leyenda que dice que } \\
\text { la aplicación se detuvo por favor arreglen eso por que es una pena no se como le siguen } \\
\text { dando propaganda en las estaciones si no funciona como la gente }\end{array}$ \\
\hline Modificabilidad & $\begin{array}{l}\text { Es muy buena idea y venia funcionando bien hasta la semana pasada (mediados de marzo). } \\
\text { Ahora las notificaciones por GPS son molestas y aunque en las opciones las desactives si- } \\
\text { guen apareciendo. Fixeen eso por favor. Otro detalle, en Motog5 plus, no aparece el servicio } \\
\text { Haedo-Temperley del FC. Roca. He... }\end{array}$ \\
\hline Utilidad & Muy util. Una lástima que no esté el ramal Belgrano Norte. Alguien sabe por qué no está? \\
\hline Confianza & la aplicacion no esta mal pensada, pero como el servicio de trenes es un desastre y no cum- \\
ple nunca jamas un horario no sirve.
\end{tabular}

Aerolíneas Móvil.

Completitud Funcional Pésima la AP. Quiero hacerme socio de aero plus y no podes desde la AP, y si queres cargar millas hay destinos que no se encuentran, como por ejemplo Río Cuarto. Igual de mala que la pagina, imposible llenar los campos de Aero Plus desde el cel, se corren y no podes completar. Todo bien con la aerolínea de bandera, pero de la primera forma que se defiende es brindandole practicidad al pasajero, que es lo mas importante que tienen.

\begin{tabular}{ll}
\hline Correctitud Funcional & $\begin{array}{l}\text { No permite agregar el vuelo comprado para chequear el estado como apps de Latam o } \\
\text { Avianca. Solo permite el vuelo un día antes. }\end{array}$ \\
\hline Eficiencia & No puedo hacer un simple check-in, se queda esperando por siempre \\
\hline Utilidad & $\begin{array}{l}\text { malisima, querés comprar el pasaje y te salta a internet. para eso busco por google y no me } \\
\text { instalo la app cracks }\end{array}$ \\
\hline Confianza & Mala aplicacion!! Resulta mas costosos los vuelos que las paginas de venta! cuando confir- \\
& mas datos resulta que no tienen itinerario!! Una verguenza! \\
\hline Aprendibilidad & la app es realmente muy mal. Está a años luz de las apps de otras aerolineas. no me mues- \\
& tra de forma automatica los pasajes que estan comprados y tienen mi codigo de viajero fre- \\
& cuente \\
\hline Estética de Interfaz & mediocre. No se qué actualizan pero parece una app de los principios de Android. Solo sieve \\
& para redireccionarte a la pagina web
\end{tabular}

MADHEL.

Completitud Funcional
Aún Faltan los LAD, pero se incorporó los procedimientos VOR, ILS, etc y planos de aeródromos lo cual es excelente. Faltaría un ingreso con CAD para enviar plan de vuelo, eso sería para aplaudir

\begin{tabular}{|c|c|}
\hline Eficiencia & Estimados, la app no termina de abrir y se cierra \\
\hline Disponibilidad & no anda se cierra sola \\
\hline Capacidad de Instalación & $\begin{array}{l}\text { No se puede actualizar el paquete mapri, sale un mensaje de "la versión del servidor se en } \\
\text { cuentra expirada" espero puedan solucionarlo a la brevedad. }\end{array}$ \\
\hline Confianza & $\begin{array}{l}\text { La versión anterior no me permitía actualizar los datos. Hoy ya puedo entrar y ver la última } \\
\text { actualización. Se ve muy util con el MAPRI y ver todos los procedimientos. Pero si deberian } \\
\text { actualizar los datos. Hay aeródromos con pista de asfalto o cemento y todavía figuran } \\
\text { como de tierra. Hay aeródromos qye existen y se vuela y no figuran. Ej. 《ITA Las Lomitas》 }\end{array}$ \\
\hline
\end{tabular}


Mi Próximo Colectivo.

Completitud Funcional Le falta desarrollo. El simple hecho de que no sirve para el propósito que fue creado hace olvidable el uso de esta app.

\begin{tabular}{ll}
\hline Correctitud Funcional & Siempre dice aguarde que haya colectivos sobre el recorrido.. nunca se actualiza \\
\hline Interoperatividad & $\begin{array}{l}\text { Muy mala aplicación me dice active GPspara mejor ubicación si tengo activo mi Gps sigue } \\
\text { el mismo cartel no me informa de las parada real }\end{array}$
\end{tabular}

Eficiencia Muy mala oscupa mucho espacio y no tiene toda las parada.

Disponibilidad Un desastre la aplicacion, ya nisiquiera funciona el sitio. Despues de cambiarle el mapa de google maps a bing, ahora ni mapa pone siquiera, dejando a la aplicacion completamente inutil. Hagan un favor y retirenla de la play store.

\begin{tabular}{ll}
\hline Capacidad de Instalación & Muy mala, estaba mucho mejor la version anterior. \\
\hline Utilidad & No sirve de nada. No info de paradas ni de cuando llega el.proximo colectivo. Una mentira \\
\hline Confianza & $\begin{array}{l}\text { mala mala. mil veces mejor la anterior. los colectivos de volcán trapiche y potrero y juana k. } \\
\text { ni aparecen. una lástima que no este la otra versión }\end{array}$ \\
\hline
\end{tabular}

BA Taxi.

Completitud Funcional No figura la puntuación del taxi, estaria bueno saberla antes de aceptar el viaje. En el mapa figura un rato y luego desaparece no sabes si esta viniendo o no. Quiero dejar de usar easy taxi pero a esta app le falta mejorar bastante.

\begin{tabular}{ll}
\hline Interoperatividad & $\begin{array}{l}\text { Imposible hacerla funcionar. Me pide que active el gps, cuando el mismo ya está activado. Y } \\
\text { de ahí no puedo pasar. }\end{array}$ \\
\hline Disponibilidad & $\begin{array}{l}\text { acabo de bajarla. Después de muchas vueltas, logre registrarme. Después me sale un men- } \\
\text { saje diciendo que necesito la última versión??? Si la acabo de bajar!!! No sirve para nada!! }\end{array}$ \\
\hline Modificabilidad & $\begin{array}{l}\text { Muy buen servicio, pero desde aproximadamente el } 19 \text { de Marzo'19 no funciona, aparece le- } \\
\text { yenda que debe ser actualizada, lo hice en mas de } 15 \text { veces y continua bloqueada, no se } \\
\text { puede usar. que paso??? 03 de Abril continúa bloqueada - los taxis deben tener mucho } \\
\text { trabajo ya que no les importa el servicio buscare otra empresa }\end{array}$ \\
\hline Capacidad de Instalación & BATAXI conDUCTOR NO SE PUEDE BAJAR DESDE UN LUMIA \\
\hline Utilidad & no me muestra por donde está viniendo el taxi. es mejor Easy Taxi \\
\hline Aprendibilidad & Me ayudan por favor, lo. Tengo tildado en el perfil, no. Me deja usar el app, ya que no me \\
& toma el número de celular \\
\hline
\end{tabular}

\section{Cómo llego.}

Completitud Funcional es bastante preciso en los viajes. Faltaría poner el tiempo de espera de los medios de transporte

\begin{tabular}{ll}
\hline Correctitud Funcional & No carga la ubicación de uno mismo. \\
\hline Disponibilidad & que pasa que no esta funcionando quiero buscar recorridos en colectivos y no deja. \\
\hline Utilidad & $\begin{array}{l}\text { Hasta la ultima actualizacion funcionaba perfectamente, pero ahora ni siquiera reconoce las } \\
\text { direcciones de Caba que se ingresan para hacer la busqueda. Solo pone "ocurrio un error. }\end{array}$ \\
\hline Confiantar" \\
no esta funcionando. cargas la dirección de como llegar y te tira un "Reintentar". Esto hace \\
varias semanas ya. Por favor una pronta solución. Gracias \\
\hline todas las líneas tiene que decir a que hora viene el colectivo, la estimación no es real. Líneas \\
como el 36 el 160 el 15 por la noche vienen cada 1 hora o más y la app dice que no tiene in-
\end{tabular}


Confidencialidad

esta app filtra datos privados de los usuarios en tiempo real y sin cifrado, por lo que cualquier persona con acceso al trafico de datos puede saber dónde está quien lo usa, que escribe y cómo. RECOMIENDO DESINSTALARLA

\section{BA Subtes.}

Completitud Funcional

La info que brinda se consigue mejor por google, deberian almenos poner los horarios, y ademas que al tocar en alguna estación aparte de la Info q ya tiene, diga cuanto falta para el Proximo subte, esas son cosas que uno necesita cuando esta en la calle. Para saber si estan funsionando solamente, no me sirve de nada.

\begin{tabular}{|c|c|}
\hline Correctitud Funcional & $\begin{array}{l}\text { Terrible la última actualización, de por si la aplicación no es muy buena ya que el buscador } \\
\text { es inútil, sin embargo el Estado de las líneas medio lo compensa, lo único útil de verdad es } \\
\text { la imagen del subte, pero ahora la app se queda colgada como } 3 \text { minutos para abrir. Hagan } \\
\text { bien su laburo o o al menos no dañen lo que no está dañado }\end{array}$ \\
\hline Eficiencia & $\begin{array}{l}\text { La aplicación simplemente se cierra sola. Mi teléfono es de última generación así que no es } \\
\text { problema de ram o cosas por el estilo. Cuando no se cerraba no devolvía resultados tan } \\
\text { simples como ir de retiro a constitucion..no la recomiendo }\end{array}$ \\
\hline Disponibilidad & $\begin{array}{l}\text { Buenas. No puedo abrir la aplicación ni reinstalando, no me acuerdo si la actualice hace } \\
\text { poco, sí del sistema hace como una semana pero nunca me fijé la app hasta ahora. Xperia } \\
\text { XZ Android } 7.1 .1\end{array}$ \\
\hline Modificabilidad & $\begin{array}{l}\text { Buenas. No puedo abrir la aplicación ni reinstalando, no me acuerdo si la actualice hace } \\
\text { poco, sí del sistema hace como una semana pero nunca me fijé la app hasta ahora. Xperia } \\
\text { XZ Android } 7.1 .1\end{array}$ \\
\hline Capacidad de Instalación & $\begin{array}{l}\text { tengo android } 9 \text { y no sirve para mi version de android! Ni que hubiera salido hace una sema- } \\
\text { na, actualicen la version! }\end{array}$ \\
\hline Utilidad & $\begin{array}{l}\text { Pensé que iba a tener la ubicación en tiempo real de las formaciones como la app de trenes } \\
\text { Argentinos. Así como está no tiene mucha utilidad, es la misma info que puedo ver en algún } \\
\text { portal de noticias sin bajar ésta app. }\end{array}$ \\
\hline Confianza & $\begin{array}{l}\text { No sirve para nada. No hay horarios en cada estación, y menciona en el caso del Premetro } \\
\text { que hay wifi, escalera mecánica y demás que son mentiras. La única estrella que indico } \\
\text { quiere decir } 0 .\end{array}$ \\
\hline Confidencialidad & ¿Por qué piden permiso para acceder al almacenamiento del teléfono? \\
\hline Aprendibilidad & jastante dificil de entender, sumado a la mala señalización. todo mal. \\
\hline Estética de Interfaz & $\begin{array}{l}\text { Muy mala interfaz. No aclara horarios, es muy mala para fijar combinaciónes. Lo único posi- } \\
\text { tivo es que tiene un Link de reclamos. Estaría bueno usarlo todos los dias ya que el servicio } \\
\text { es pésimo y poder quejarte de esa forma. }\end{array}$ \\
\hline
\end{tabular}

BA Turismo.

Completitud Funcional Buena app para encontrar lugares que visitar, aunque le falta incluir más sitios especificos e incluir un mapa de la ciudad.

Correctitud Funcional Es muy interesante, tiene buenas cosas y guías. Pero funciona mal, parece como incompleta o con bugs

Capacidad de Instalación Quise instalarlo y me da error en la descarga e instalación. o sea, no puedo siquiera instalarla.

Utilidad No puedo hacer ninguna reservas. Es posible que todas las que intentó reservar ya no hay lugares disponibles..? Sólo sirve para ver que hay. 


\begin{tabular}{ll}
\hline Aprendibilidad & Muy buena idea y de gran ayuda para el turista. No le pongo 5 estrellas por la poca cantidad \\
& y baja calidad de las imágenes y por no encontrar como ver las opiniones del resto de los \\
& usuarios. Otra opción que podría agregarse es una audioguia que pueda descargarse para \\
& realizar los tour por fuera de los horarios ofrecidos.
\end{tabular}

\section{Ciudad de Mendoza.}

Completitud Funcional Hace tres semanas llegue a Mendoza para quedarme a vivir, que pena que esta app no tiene los recorridos de los micros.... y si los tiene no los puedo ver porque no funciona la búsqueda por voz

\begin{tabular}{|c|c|}
\hline Correctitud Funcional & la baje para sacar turnos eso decia la pag y no sirve para sacar turno \\
\hline Eficiencia & Re lenta. Parece exploret. \\
\hline Disponibilidad & $\begin{array}{l}\text { Muy mala app. No es efectiva. Demora mucho en cargar. La agenda cultural solo muestra } \\
\text { los eventos pasados y el evento del día de hoy (para qué quiero saber qué hubo hace } 1 \text { mes, } \\
\text { ¿no creen que es más útil difundir la agenda cultural de los próximos días?). El botón de pá- } \\
\text { nico parece una tomada de pelo, está mal diseñado, se quedaron a mitad de camino. Ya } \\
\text { mismo la desinstalo. \#DECEPCIÓN }\end{array}$ \\
\hline Utilidad & no encuentro donde sacar turno para la licencia de conducir \\
\hline Confianza & $\begin{array}{l}\text { no tiene informacion actulaizada, algo tan simple como cargar un cronograma no lo hacen } \\
\text { no hacen nada bien, me pregunto a cuantos inutiles se les paga por esto, asi esta la ciudad } \\
\text { es pura pinta a la hora de solucionar algo un mono lo hace mucho mejor }\end{array}$ \\
\hline Aprendibilidad & $\begin{array}{l}\text { Cultura Mendoza Hola donde puedo encontrar la app o en esta app de la agenda cultural } \\
\text { mendoza?? }\end{array}$ \\
\hline Estética de Interfaz & $\begin{array}{l}\text { Muy buena app me gustó bastante aunque falta mejorar la interfaz en si del menú muchas } \\
\text { gracias }\end{array}$ \\
\hline
\end{tabular}

Ushuaia Turismo.

Completitud Funcional Sólo sirve para ubicar lugares ya que no tiene ninguna descripción ni información de las actividades y demás cosas.

Disponibilidad Toda la info oficial de Ushuaia Funciona muy bien en Samsung Note 8 , le doy 4 * porque sólo funciona con conexión de datos o wi fi. Por lo demás, va muy bien con el gps del celu y la info es muy buena, recomendable para cualquier viajero

Utilidad la app muestra sólo posibilidades pagas. nada para el turista gasolero! No da información más que de los Tours que son carísimos!

\section{Turismo Santa Fe Ciudad.}

Completitud Funcional Esta bien. Pero muy pobre el diseño. Una lista interminable. Faltaría agregar la agenda de eventos de la ciudad. Pero bien.

Disponibilidad no funciona

Neuquén Guía de Viaje.

Completitud Funcional Camping Es un dato faltante. No hay información para acampar con carpas o rodantes

Eficiencia Bueno Que tal, estaría bueno que en la ciudades que tiene buena conexión no descargue to.
dos los mapas por defecto así se reduciría el peso de la misma. Saludos

Confianza Falta info Muy poca información de alojamiento y datos en general. Le falta mucho todavía!

\footnotetext{
Alertamos.

Completitud Funcional Un gráfico con detalles que cite un informe de temperatura si Sta soleado, ventoso velocidad del viento etc sería genial.
} 


\begin{tabular}{|c|c|}
\hline Eficiencia & $\begin{array}{l}\text { Podrían mejorar, xq se tilda y no puede verse bien, es pregunté a mejor usar la página y no la } \\
\text { app }\end{array}$ \\
\hline Disponibilidad & Solia ser una buena app para el clima en Argentina, pero ahora ni funciona \\
\hline Modificabilidad & $\begin{array}{l}\text { Me decepcionó. Anteriormente yo la había descargado en otro celular (Motorola X Style } \\
\text { Bambú XT1572) y me funcionaba excelente, ahora tengo otro celular (LG G5), y la volví a } \\
\text { descargar para estar informado sobre el clima, peroo...me decepcionó, no funciona, abre } \\
\text { pero el mapa nunca carga, queda en blanco y jamás detecta mi ubicación, cuando en la app } \\
\text { Maps de Google si me detecta la ubicación a la perfección... Espero me den una solución } \\
\text { pronto o perderán un Usuario. }\end{array}$ \\
\hline Capacidad de Instalación & Solia ser una buena app para el clima en Argentina, pero ahora ni funciona \\
\hline Utilidad & La aplicación no debería estar publicada. No muestra los mapas ni alertas. \\
\hline Confianza & $\begin{array}{l}\text { bueno seria que muchos la bajen y principalmente cuando hay una evento de lluvia, viento, } \\
\text { granizo, etc los publiquen, entre todo es mejor. }\end{array}$ \\
\hline Aprendibilidad & $\begin{array}{l}\text { La idea está, pero le falta mucho. Pensé q se podría ver el clima a todo momento y no solo } \\
\text { con los reportes. No me registra mi ubicación. Y no me permite marcar más de } 1 \text { fenómeno } \\
\text { a la vez, llovía y caía granizo si marcaba una no podía alertar sobre la otra... mucho por mejo- } \\
\text { rar }\end{array}$ \\
\hline Estética de Interfaz & $\begin{array}{l}\text { Muy fea la aplicación, la hicieron con dos pesos. Se ve muy fea parece que es para windows } \\
98 \text {... no carga y se tilda, no me gusta }\end{array}$ \\
\hline
\end{tabular}

Museo Nacional del Cabildo.

Eficiencia

Me dio virus

Aprendibilidad Pesada y aburrida

Estética de Interfaz Poco interactivo. Pense que tenia recorrido en 3D. Parece una app de principios del 2000

\section{Radio Nacional Argentina.}

Completitud Funcional Cada filial tiene 2 emisoras. Estaria bueno que solo aparezca en la app la emisora que tenga la programacion local, casi siempre la AM. Sería mas facil encontrar el programa que quiero escuchar

\begin{tabular}{ll}
\hline Eficiencia & tastidio total , se interrumpe a cada rato!!!! siempre tiene que ser pesimo lo del estado!!!!! \\
\hline Disponibilidad & Se desconecta continuamente \\
\hline Modificabilidad & $\begin{array}{l}\text { Antes funcionaba Después de actualizar no funciona en absoluto. Es una lástima, me en- } \\
\text { cantaba este app. }\end{array}$ \\
\hline Utilidad & No funciona la It 11 c.del Uruguay \\
\hline Confianza & $\begin{array}{l}\text { Es muy lenta además estãn mal los nombres Los nombres no corresponden a algunas } \\
\text { emisoras. Ej Malargüe corresponde am } 790 \text { y no AM } 620 \text { de San Rafael. }\end{array}$ \\
\hline
\end{tabular}

\section{SIF Móvil Min. Agroindustria.}

\begin{tabular}{ll} 
Completitud Funcional & $\begin{array}{l}\text { Sería bueno poder usarla desde PC y poder consultar faenas anteriores en un resumen y po- } \\
\text { der bajar la información. }\end{array}$ \\
\hline Correctitud Funcional & $\begin{array}{l}\text { Cuando lo trate de usar a mi nunca me funciono lamentablemente, mande mail a los que la } \\
\text { hicieron en varias ocasiones y jamas me respondieron, ne gustaria saber la razon por lo cual } \\
\text { me dice q el dte no existe cuando toda la información q cargo es correcta }\end{array}$ \\
\hline Aprendibilidad & Buen dia, la aplicacion funciona pero no me ofrece los datos detallados de romaneo. Que \\
& puedo hacer?
\end{tabular}

